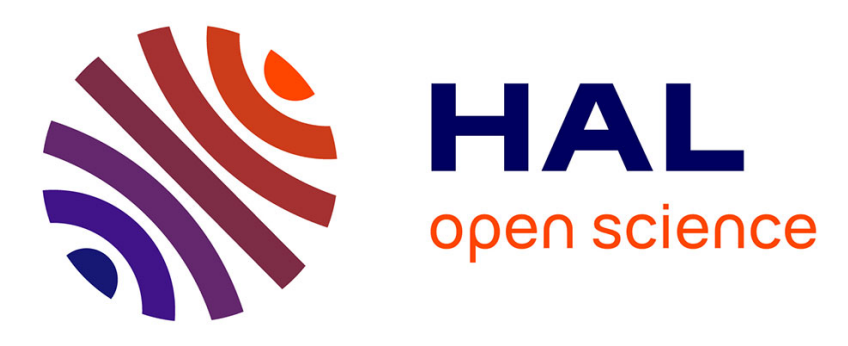

\title{
Saturation dependence of the streaming potential coefficient
}

Laurence Jouniaux, Vincent Allègre, Renaud Toussaint, Fabio Zyserman

\section{To cite this version:}

Laurence Jouniaux, Vincent Allègre, Renaud Toussaint, Fabio Zyserman. Saturation dependence of the streaming potential coefficient. Seismoelectric Exploration: Theory, Experiments, and Applications, Wiley, pp.73-100, 2020, 9781119127376. 10.1002/9781119127383.ch5 . hal-03015990

\section{HAL Id: hal-03015990 https://hal.science/hal-03015990}

Submitted on 20 Nov 2020

HAL is a multi-disciplinary open access archive for the deposit and dissemination of scientific research documents, whether they are published or not. The documents may come from teaching and research institutions in France or abroad, or from public or private research centers.
L'archive ouverte pluridisciplinaire HAL, est destinée au dépôt et à la diffusion de documents scientifiques de niveau recherche, publiés ou non, émanant des établissements d'enseignement et de recherche français ou étrangers, des laboratoires publics ou privés. 


\section{Saturation dependence of the streaming potential coefficient}

${ }^{1}$ Université de Strasbourg, CNRS, Institut de Physique du Globe de Strasbourg UMR7516, 5 rue René Descartes, 67084, Strasbourg, France. ${ }^{2}$ Université de Bordeaux, Institut de Mécanique et d'Ingénierie, Département Génie Civil et Environnemental, CNRS UMR 5295, 33615

${ }^{3}$ Facultad de Ciencias Astronómicas y Geofísicas, Universidad Nacional de La Plata, Paseo del Bosque s/n, B1900FWA La Plata, Argentina ${ }^{4}$ CONICET

\footnotetext{
*vincent.allegre@u-bordeaux.fr

† toussaint@unistra.fr

${ }^{\ddagger}$ zyserman@fcaglp.unlp.edu.ar
}

Corresponding author: L. Jouniaux, 1 . jouniauxeunistra.fr 
Observations of streaming potential for unsaturated conditions do not always show the same trend depending on the hydrodynamic conditions and because of a lake of coherency between the data processing procedures. We combine the data from three studies published in the literature, acquired during non-steady state drainage experiments, and apply the same processing steps. We model the hydrodynamic behaviour of these experiments to confirm that they experienced different flow dynamics. We argue that the raw SP data should not be corrected unless a clear drift of the electrodes stability is observed. The combined hydrodynamic behaviour and the streaming potential response show that (a) the observations of one of the experiment (exp \#1) are associated to a limited range of water saturation (0.85-1). The corresponding signals could therefore be fairly modelled assuming no saturation dependence of the SPC whatsoever; (b) the observations of exp \#3 led to a SPC that can be larger than its value at saturation; (c) the observations of the exp \#2 show a non-monotonous behaviour of the SPC as saturation decreases. The underlying physics of a non-monotonous $\mathrm{SPC}$ is related to water/air interfaces as shown by the results of the lattice Boltzmann numerical simulations. The main contribution to the SPC behaviour comes from the charged water/air interfaces and depends on the dynamic state of moving or entrapped bubbles. We finally describe the consequences of such a behaviour on the seismoelectric conversions for unsaturated conditions.

\section{Introduction}

Electrokinetics are at the origin of geophysical methods used to investigate the near-surface earth system in terms of fluid transfers. Both seismoelectrics and most of self-potentials observations are interpreted to be induced by a relative motion between the rock matrix and the saturating fluid, converted into an electric field and a magnetic field through electrokinetic coupling.

Seismoelectrics and electroseismics were developed to investigate hydrogeological reservoirs [Dupuis et al., 2007; Dupuis et al., 2009; Schakel et al., 2012; Warden et al., 2013], specially in arid environments [Valuri et al., 2012], and hydrocarbon reservoirs [Thompson et al., 2005; Hornbostel and Thompson, 2007; Hu et al., 2007; Thompson et al., 2007; Zyserman et al., 2012; Guan et al., 2013; Zyserman et al., 2015]. More- 
over numerical, experimental and field studies have also investigated borehole geometries [Hunt and Worthington, 2000; Hu and Liu, 2002; Dupuis et al., 2009; Wang et al., 2015a] and possible applications for the vadose zone [Zyserman et al., 2017a,b; Monachesi et al., 2017]. The reader can find more details with many references in the large review from Jouniaux and Zyserman [2016]. Some other works studied the seismo-electromagnetic signals that could be induced by earthquakes [Matsushima et al., 2002; Gaffet et al., 2003; Guglielmi et al., 2006; Ren et al., 2010; Gao et al., 2013, 2016].

Most of the works were developed considering a single saturating fluid at full saturation. It was only in the few last years that attempts to introduce partial saturation in seismoelectrics/electroseismics have been undertaken [Warden et al., 2013; Zyserman et al., 2015]. Besides of using different known effective media models for the different physical parameters of the seismoelectrics/electroseismic equations, the key model parameter, i.e., the electrokinetic coupling, has been modified to cope with partial saturations following the ideas previously used to extend the streaming potential coefficient (SPC) to handle this situations.

Self-potentials rely on electrokinetic coupling and proved to be efficient for characterizing groundwater resources and mapping contamination plumes, since those signals are directly sensitive to hydrological fluxes [Fournier, 1989; Titov et al., 2002; Pinettes et al., 2002; Maineult et al., 2004; Jouniaux et al., 2009; Desroches and Butler, 2016] and solute transport [Maineult et al., 2005; Kabir et al., 2015; Giampaolo et al., 2016]. Self-potentials appeared especially useful to estimate fluid flow [Jouniaux et al., 1999; Sailhac et al., 2004; Rangarajan et al., 2014], to detect propagating vapor-front during oil-extraction processes [e.g., Saunders et al., 2008], to detect hydrothermal flows [Mauri et al., 2010; Brothelande et al., 2014; Gonzales et al., 2014], to map advected reacting redox fronts [Maineult et al., 2006a,b], and to provide a tool to evaluate the evolution of the corrosion of metallic casings [Maineult, 2016]. Self-potentials are also often associated with natural hydrothermal circulation [Ishido and Pritchett, 1999; Matsushima et al., 2000; Jouniaux and Ishido, 2012; Monetti et al., 2014]. The knowledge of the electrokinetic coupling is therefore significant to the interpretation of a broad range of geophysical methods.

Many experimental studies on streaming potential measurements in porous samples have been published in the past three decades [Wang et al., 2015b; Walker et al., 2014; Luong and Sprik, 2014; Glover et al., 

2006; Perrier and Froidefond, 2003; Hase et al., 2003; Guichet et al., 2003; Jouniaux et al., 2000; Lorne et al., 1999; Pozzi and Jouniaux, 1994; Jouniaux et al., 1994; Ishido and Mizutani, 1981].

A few experimental studies addressed the SP (self-potential) behaviour associated with unsaturated flow. Early observations performed during unsaturated water flow experiments on sand showed that SP amplitudes decrease at smaller water content [Guichet et al., 2003], which was supported by some theoretical models [e.g., Perrier and Morat, 2000; Revil et al., 2007]. Linde et al. [2007] proposed similar SP measurements that were also coherent with the existing model by Revil et al. [2007].

The first SPC measurements performed as a function of continuously decreasing water saturation were reported by Allègre et al. [2010]. The raw SPC curves unexpectedly showed a non-monotonic shape as water content decreases, which could not be described by any existing models at the time. Revil and Linde [2011] argued that unconsistent signal processing as well as experimental issues regarding electrode stability were responsible for Allègre et al. [2010] conclusions, which was demonstrated to be very unlikely [Allègre et al., 2011]. Allègre et al. [2012] then supported Allègre et al. [2010] observations with a combined modelling of the hydrodynamic conditions and the electrical potential simulating the same drainage experiments.

The influence of electrode stability on unsaturated SP measurements has to be treated carefully and was investigated by Jougnot et al. [2012] who proposed that a drifting correction should be applied to raw SP measurements, especially at low water saturation. Another kind of experimental correction was used by Mboh et al. [2012] to address electrode drifting. Allègre et al. [2014] proposed continuous self-potential measurements performed during drainage and imbibition cycles. After demonstrating that electrodes were extremely stable over time, especially at very low water saturation, they concluded that none of the existing SPC models could correctly describe their observations. A semi-empirical expression addressing the drainage/imbibition cycles dynamics was used to model the SP measurements. The flow dynamics and the behaviour of water/air interfaces were argued to be of significant influence on the response of unsaturated SP [Allègre et al., 2015].

Few theoretical and numerical studies showed that large values of the SPC can be observed under specific conditions regarding the chemistry of the electrolyte and structural properties of the porous medium [e.g., 
Jackson, 2008, 2010]. Numerical simulations performed by Zhang et al. [2014] suggested that the SPC exhibits an hysteresis between drainage and imbibition and that it can vary non-monotonically with saturation. Up to this date, neither existing datasets, nor numerical models are entirely consistent.

The current contribution aims to reconcile the experimental SP data from Linde et al. [2007], Mboh et al. [2012] and Allègre et al. [2010] by using the same processing steps. We emphasise that the water/air interfaces have to be included in the modelling process so that it can properly describe the unsaturated SP response.

\section{State of the art}

We present in this section the theoretical background of the electrokinetics and particularly of the streaming potential coefficient. We describe the different theories and models of the streaming potential as a function of saturation. Then we show measurements of the streaming potential coefficient as a function of the water-saturation.

\subsection{Theoretical background}

The electrokinetic effect is due to fluid flow in porous media because of a particular distribution of ions within the electric double layer, which can induce a streaming electric current when water flows, which is counterbalanced by a conduction current. The general equation coupling the different flows is:

$$
\mathbf{J}_{i}=\sum_{j=1}^{N} \mathcal{L}_{i j} \mathbf{X}_{j}
$$

which links the forces $\mathbf{X}_{j}$ to the macroscopic fluxes $\mathbf{J}_{i}$, through transport coupling coefficients $\mathcal{L}_{i j}$ [Onsager, 1931]. Notice that boldface is used to denote vector quantities.

In seismoelectrics the coupling between the hydraulic flow and the electric flow is induced by seismic wave propagation, which leads to a relative motion between the fluid and the rock matrix. The theory for the coupled electromagnetics and acoustics of porous media was developed by Pride [1994]. The trans- 
port relations (Pride, 1994, Eqs. 250 and 251) are:

$$
\begin{aligned}
& \mathbf{J}_{\mathrm{e}}=\hat{\sigma}(\omega) \mathbf{E}+\hat{\mathcal{L}}(\omega)\left(-\nabla P+\omega^{2} \rho^{\mathrm{f}} \mathbf{u}_{\mathrm{s}}\right), \\
& \mathbf{J}_{f}=\hat{\mathcal{L}}(\omega) \mathbf{E}+\frac{k(\omega)}{\eta}\left(-\nabla P+\omega^{2} \rho^{\mathrm{f}} \mathbf{u}_{\mathrm{s}}\right),
\end{aligned}
$$

where an $e^{-i \omega t}$ time dependence has been assumed. The electrical fields and mechanical forces that induce the electric current density $\mathbf{J}_{\mathrm{e}}$ and the fluid volume flux $\mathbf{J}_{\mathrm{f}}=-i \omega \mathbf{w}_{f}$ are, respectively, $\mathbf{E}$ and $(-\nabla P+$ $i \omega^{2} \rho^{\mathrm{f}} \mathbf{u}_{\mathrm{s}}$ ), where $P$ is the pore-fluid pressure, $\mathbf{u}_{\mathrm{s}}$ and $\mathbf{w}_{f}$ are respectively the solid and fluid displacements, $\mathbf{E}$ is the electric field, $\rho^{\mathrm{f}}$ is the pore-fluid density, $\eta$ the dynamic viscosity of the fluid [Pas], and $\omega$ is the angular frequency.

The electrokinetic coupling $\left.\hat{\mathcal{L}}_{(} \omega\right)$ describes the coupling between the seismic and electromagnetic fields and is complex and frequency-dependent [Pride, 1994; Reppert et al., 2001]:

$$
\hat{\mathcal{L}}(\omega)=\mathcal{L}_{0}\left[1-i \frac{\omega}{\omega_{c}} \frac{m}{4}\left(1-2 \frac{d^{l}}{\Lambda}\right)^{2}\left(1-i^{3 / 2} d^{l} \sqrt{\frac{\omega \rho^{\mathrm{f}}}{\eta}}\right)^{2}\right]^{-\frac{1}{2}},
$$

where $m$ and $\Lambda$ are geometrical parameters of the pores ( $\Lambda$ is defined in Johnson et al. [1987] and $m$ is in the range 4-8), $d^{l}$ the Debye length. The electrokinetic coupling is an important parameter: if this coupling is zero, then there is no seismo-electric nor electro-seismic conversion.

The electrokinetic coupling is related to the streaming potential coefficient $\hat{C}_{\mathrm{s}}$ as

$$
\hat{\mathcal{L}}(\omega)=-\hat{\sigma}(\omega) \hat{C}_{\mathrm{s}}(\omega)
$$

with $\sigma$ the rock conductivity. The frequency-dependence of the streaming potential coefficient [Jouniaux and Bordes, 2012] can be measured as the ratio between the electric field induced by an applied pressure gradient, when the electric current density $\mathbf{J}_{\mathrm{e}}$ is zero as:

$$
\hat{C}_{\mathrm{s}}(\omega)=\frac{\Delta V(\omega)}{\Delta P(\omega)}
$$

This coefficient has been studied on synthetic samples [Packard, 1953; Cooke, 1955; Groves and Sears, 1975; Sears and Groves, 1978; Chandler, 1981; Reppert et al., 2001; Schoemaker et al., 2007, 2008], and recently on sand [Tardif et al., 2011], and on unconsolidated materials [Glover et al., 2012]. However the effect of 
The steady-state electrokinetic coupling is defined as:

$$
\mathcal{L}_{0}=-\sigma C_{0}\left(1-2 \frac{\tilde{d}}{\Lambda}\right)
$$

where the streaming potential coefficient $C_{0}$ (or SPC) $\left(\mathrm{PPa}^{-1}\right)$ is defined when the electric current density $\mathbf{J}_{\mathrm{e}}$

$$
\mathbf{J}_{\mathrm{e}}=-\sigma \nabla V-\mathcal{L}_{0} \nabla P
$$

is zero.

When the surface conductivity can be neglected compared to the fluid conductivity, and assuming a laminar fluid flow and identical hydraulic and electric tortuosity, the streaming coefficient is described by the well-known Helmholtz-Smoluchowski equation [e.g., Dukhin and Derjaguin, 1974]:

$$
C_{0}=\frac{\Delta V}{\Delta P}=-\frac{\mathcal{L}_{0}}{\sigma}=S P C=\frac{\epsilon_{\mathrm{f}} \zeta}{\eta \sigma^{\mathrm{f}}}
$$

The relevant parameters influencing the SPC are the fluid relative permittivity $\epsilon_{\mathrm{f}}$, the fluid viscosity $\eta$, the fluid conductivity $\sigma^{\mathrm{f}}$ and the zeta potential $\zeta$, itself depending on rock, fluid composition, and $p H$. The effect of these parameters has been modeled using Lattice-Boltzmann simulations within a large range of salinity and compared to measured SPC [Fiorentino et al., 2016].

Depending on the type of rocks the surface conductivity may not be negligible as for clay for example. Then the streaming potential coefficient can be written as:

$$
C_{0}=\frac{\epsilon_{\mathrm{f}} \zeta}{\eta\left(\sigma^{\mathrm{f}}+\sigma_{\mathrm{s}}\right)},
$$

with $\sigma_{\mathrm{s}}$ the surface conductivity $\left(S \mathrm{~m}^{-1}\right)$ which can be inferred from $\sigma_{\mathrm{s}}=2 \Sigma_{\mathrm{s}} / R$, with $R$ the hydraulic radius of the rock or the pore radius and $\Sigma_{\mathrm{S}}$ the surface conductance (S) [Rutgers, 1940; Alkafeef and Alajmi, 2006; Wang and $\mathrm{Hu}, 2012]$. In Fontainebleau sand $\sigma_{\mathrm{s}}$ is less than $2 \times 10^{-4} \mathrm{Sm}^{-1}$ [Guichet et al., 2003]. For quartz or sandstone typical values of $\Sigma_{\mathrm{S}}$ range from $8.9 \times 10^{-9}$ to $4.2 \times 10^{-8} \mathbf{S}$ [Block and Harris, 
2006] and $2.5 \times 10^{-9} \mathbf{S}$ for clays [Revil and Glover, 1998]. The surface conductivity can neither be neglected in clay layers, nor when the hydraulic radius is of the order of the Debye length. This latter case can be encountered when the fluid is not very conductive, as below $2 \times 10^{-3} \mathrm{~S} \mathrm{~m}^{-1}$ in sandstones [Pozzi and Jouniaux, 1994]. In that case the streaming potential coefficient can be lowered compared to the expected value. Since the hydraulic radius can be indirectly connected to the permeability, the effect of surface conductivity can explain some observations of permeability-dependence of the streaming potential coefficient [Jouniaux and Pozzi, 1995].

Another way to take into account $\sigma_{\mathrm{s}}$ without knowing the surface conductivity nor the conductance [Jouniaux et al., 2000], but knowing the rock conductivity $\sigma$ possibly with a surface component and the formation factor $F$ is:

$$
C_{0}=\frac{\epsilon_{\mathrm{f}} \zeta}{\eta \sigma_{\mathrm{eff}}}=\frac{\epsilon_{\mathrm{f}} \zeta}{\eta F \sigma}
$$

\subsection{Streaming potential coefficient modelling for unsaturated conditions}

The effect of water-saturation on the SPC is still discussed, both through modelling and through the interpretation of the observations, although the effect of saturation on electrical properties is well known [Archie, 1942; Carrara et al., 1999; Tsakiroglou and Fleury, 1999; Zhou et al., 2002; Bekri et al., 2003; Jouniaux et al., 2006; Kim et al., 2013]. Most of the models for the SPC can be written as a product between the SPC at full saturation $C_{\text {sat }}$ and a function of saturation $S\left(S_{w}\right)$ as

$$
C_{0}\left(S_{w}\right)=C_{s a t} S\left(S_{w}\right)
$$

Perrier and Morat [2000] were the first to propose that the SPC depends on the relative permeability, and proposed that SPC depends on the relative permeability $k_{\mathrm{r}}$ as:

$$
C_{0}\left(S_{w}\right)=C_{\mathrm{sat}} \frac{k_{\mathrm{r}}\left(S_{w}\right)}{S_{w}^{n}}
$$

with $S_{w}$ the water saturation, and $n$ the second Archie exponent [Archie, 1942], where $k_{r}$ is the relative permeability defined as: $k_{r}\left(S_{w}\right)=\left(S_{w}-0.1 / 0.9\right)^{2}$. Later Revil et al. [2007] proposed a similar formula as 
proposed by Perrier and Morat [2000], assuming that the excess countercharge density scales inversely with water saturation.

Then Jackson [2010] developed a model for the SPC for unsaturated conditions through a capillary tubes model, including water or oil as fluid. Jackson [2010] showed that the electrokinetic coefficient depends on the relative permeability, the relative charge density, and the fluid content, assuming that Archie's law is valid, as:

$$
C_{0}\left(S_{w}\right)=C_{\mathrm{sat}} \frac{k_{\mathrm{r}}\left(S_{w}\right) Q_{\mathrm{r}}\left(S_{w}\right)}{S_{w}^{n}}
$$

with $Q_{\mathrm{r}}$ the relative excess charge density: $Q_{\mathrm{r}}\left(S_{\mathrm{w}}\right)=Q\left(S_{w}\right) / Q\left(S_{w}=1\right)$. Jackson $[2008,2010]$ showed that the relative excess charge density depends on the pore-scale distribution of fluid and charge, and that it does not scale inversely with the water saturation. Jackson [2010] showed that the relative excess countercharge density increases with decreasing water saturation in water-wet models, because water is first emptied from the large capillaries. As a result, the water progressively occupies a larger amount of small capillaries. All these models describe a monotonous decrease of the SPC from $C_{s a t}$ to zero with decreasing saturation, but it has been shown that the relative $\mathrm{SPC} C_{r}$ (i.e., normalized to $C_{s a t}$ ) could be higher than 1 , taking into account the specific flow and electrolyte properties [Jackson, 2010].

Allègre et al. [2012] modelled both Richards' equation for hydrodynamics and the Poisson's equation for electrical potential for unsaturated conditions using a 1-D finite element method, which was never reported for unsaturated conditions. They concluded, based on laboratory experiments and using these equations, that the unsaturated electrokinetic coefficient should have a non-monotonic behaviour:

$$
C_{0}=C_{\mathrm{sat}} S_{\mathrm{e}}\left[1+\beta\left(1-S_{\mathrm{e}}\right)^{\gamma}\right]
$$

where the effective saturation is:

$$
S_{\mathrm{e}}=\frac{S_{w}-S_{w r}}{1-S_{w r}}
$$

and $\beta$ and $\gamma$ are two adjusted parameters, $\beta$ depending on the initial flow conditions, particularly on the water velocity at the beginning of the drainage phase. Table 1 summarises the values of $C_{0}\left(S_{\mathrm{w}}\right) / C_{\text {sat }}=S(w)$ gathered from the literature. 
The first experimental study on SP dependence on water content was carried out by Guichet et al (2003). They measured the SP associated to water flow during an experiment performed by injecting inert gas into a column filled with Fontainebleau sand ( $>99 \%$ quartz rich sand). The results, corrected from $\mathrm{pH}$ and electrical conductivity variations (Fig.1), suggested a monotonous and linear behaviour of the SPC as a function of the water saturation. They therefore proposed a linear relation, via:

$$
C_{0}\left(S_{e}\right)=C_{\text {sat }} S_{e}
$$

Linde et al. [2007] measured SP signals associated to a gravity-driven drainage experiment and reported pressure head and outflow measurements. They concluded that the SP recordings could be modelled using the model from Revil et al. [2007]. We further discuss the results from this study in section 3. Allègre et al. [2010] reported the first continuous recordings of SP measured during a drainage experiment. Two main important observations were presented: 1) the SPC can be one order of magnitude larger than its value at saturation $\left.\left(C_{s a t}\right) ; 2\right)$ its behaviour can be non-monotonic (first increasing then decreasing) with decreasing water saturation (Fig.2). The SPC reaches a maximum value that depends on the water flow velocity. No existing models could support the dataset. The non-monotonic behaviour of the SPC is partly supported by the observations from Revil et al. [2007] and Revil and Cerepi [2004] as discussed in Allègre et al. [2011], once the SPC value measured at saturation from Revil and Cerepi [2004] is properly included (Fig. 3).

Vinogradov and Jackson [2011] published SP data acquired during multiphase flow (e.g., water/air or water/oil) imbibition and drainage experiments. They used brine/undecane and brine/nitrogen mixtures. The SP measurements were provided at isolated water saturation values ranging from 0.2 to 0.5 . One measurement was provided for saturated conditions (Fig. 4), but no data points were available for saturation between 0.5 and 1. It was therefore difficult to conclude whether the SPC behaviour is non-monotonic in that case.

Mboh et al. [2012] reported streaming potential and water pressure measurements performed during a drainage experiment. The authors proposed to remove a trend from the SP data to ensure zero electrical potential difference at the end of the experiment (i.e., in no flow conditions) and the the value. The correction also guarantied the SP signal to fit eq. 13 for saturated conditions. The resulting corrected SPC curves 
were supported by the model from Revil et al. [2007]. This data set is analyzed from scratch in the following section.

Jougnot and Linde [2013] performed a few drainage and imbibition experiments providing both SP and hydrodynamic measurements. They observed some features in the SP signals that they attributed to electrode issues. They compared their data set to the one from Mboh et al. [2012] and proposed that SP data should be corrected from electrode effects such as leakage, especially when the medium is quite unsaturated. Allègre et al. [2014] utilized another apparatus to measure self-potentials signals during drainage and imbibition cycles. They argued that non-zero SP signals could occur for unsaturated conditions and proposed a water outflow based model to fit their data set. The measurements did not show any evidence for electrode leakage that proved to be extremely stable over time, even for very low saturation values. They suggested that water/air interfaces could be responsible for such variations. The influence of water/air interfaces on SP measurements is fully discussed in section 4 .

\section{Cross-analysis of streaming-potential data}

\subsection{Background}

Among the contributions on SP measurements published in the literature, we chose to combine the data from Linde et al. [2007], Allègre et al. [2010] and Mboh et al. [2012], that we refer to as experiments I, II, and III in the following. The three datasets were acquired under similar experimental conditions and exclusively relate to drainage experiments performed in sand. The raw SP and hydrodynamic data available for each study were used. We included an original dataset acquired using the experimental setup described in Allègre et al. [2014] and designed for drainage/imbibition cycles experiments. We refer to this work as experiment IV in the results section. We chose to focus on SP data recorded during non-steady state experiments, since the dynamics of the water flow is a key parameter. We therefore did not include SPC measurements performed in consolidated samples in quasi-static conditions [e.g., Revil and Cerepi, 2004; Vinogradov and Jackson, 2011] . 


\subsection{Experimental procedures}

The drainage experiment proposed by Linde et al. [2007] was performed in a $135 \mathrm{~cm}$ long and $35 \mathrm{~mm}$ wide poly-vinyl chloride tube. The medium was a fine grain quartz sand saturated with tap water, whose the electrical conductivity was $\sigma_{w}=0.051 \mathrm{~S} / \mathrm{m}$. The bulk electrical conductivity of the saturated mixture was $\sigma_{\text {sat }}=0.012 \mathrm{~S} / \mathrm{m}$. The water pressure was monitored with ten tensiometers spread along the column (Fig. 5). The SP data were measured with 17 non-polarizable electrodes. The water contents were not measured. The experimental course was described as: 1) a drainage phase was triggered by applying a pressure boundary condition of $9.1 \mathrm{~cm}$ high water column at the lower end of the column, while the upper end was open to atmosphere; 2) this lower boundary condition was removed after 6 hours, allowing a free gravity-driven drainage phase to occur.

Allègre et al. [2010] experiment consisted in draining a $1.3 \mathrm{~m}$ long and $10 \mathrm{~cm}$ wide plexiglass column filled with quartzic sand initially saturated with chemically equilibrated water. A constant pressure head of $2 \mathrm{~cm}$ was applied at the bottom of the column to drive the drainage experiment. The procedure stopped when no outflow was recorded. The water pressure heads, water contents and SP data were monitored at ten levels inside the column during the experiment (Fig. 5).

The experiment by Mboh et al. [2012] was performed in a $217.5 \mathrm{~cm}$ long and $5 \mathrm{~cm}$ wide plastic tube. The medium was also a quartz sand, compacted on a portion of the column $(117.5 \mathrm{~cm})$. The water pressure were measured using 6 tensiometers, and 10 SP electrodes were located along the column (Fig. 5). The protocol was designed in three steps: 1) the system was monitored at the hydrodynamic equilibrium (i.e., no flow occurring) for 22 minutes; 2) a Mariotte reservoir was used to apply a constant head at the top for 38 minutes; 3) the reservoir is opened allowing a falling head experiment, followed by a drainage phase of the column.

The experiment described by Allègre et al. [2014] was designed to monitor self-potential signals associated with drainage and imbibition cycles. The authors used a $30 \mathrm{~cm}$ long and $10 \mathrm{~cm}$ wide plexiglass column filled with saturated quartz sand. The singular experiment included here consisted of a step-by-step drainage phase, followed by a step-by-step imbibition phase. The pressure heads and self-potentials were measured 
at two locations along the column. The procedure consisted in incrementally decreasing the bottom pressure boundary condition to allow drainage. The imbibition phase mimics these steps by incrementally increasing the boundary condition until the initial condition was reached. The water contents were computed from solving the flow equation (details in the next section). The SP signals were measured between electrodes located along the column and a reference electrode located at the very bottom, in the saturated part of the system. Using a reference electrode remaining in the water provides a stable potential. This common reference allows to infer any dipole differences afterwards.

\subsection{Hydrodynamic modelling}

The hydrodynamic conditions of the experiments need to be constrained so that one can compute the SPC from the computed water pressure differences. Unsaturated flow through a homogeneous porous medium is well described by the Richards equation [Richards, 1931], which is formulated in 1D in its mixed form as,

$$
\frac{\partial \theta(h)}{\partial t}-\frac{\partial}{\partial z}\left[K(h)\left(\frac{\partial h}{\partial z}-1\right)\right]=0
$$

where $\theta(h)$ is the volumetric water content in $\mathrm{m}^{3} \cdot \mathrm{m}^{-3}$, depending on the pressure head $h(\mathrm{~m})$. The parameter $K$ is the hydraulic conductivity $\left(\mathrm{m} \cdot \mathrm{s}^{-1}\right), t$ is time (s), and $z$ is the vertical coordinate (m), positive downward. The retention properties and a relative permeability model are necessary to solve eq. 18 . Various models are available to assess those properties and the studies presented here rely on a few different ones.

Linde et al. [2007] used the van Genuchten [1980] model which describes the behaviour of $h$ as a function of the water content as,

$$
S_{e}=\frac{\theta-\theta_{r}}{\theta_{s}-\theta_{r}}=\frac{1}{\left(1+\left(\alpha_{v}|h|\right)^{n}\right)^{m}},
$$

where $S_{e}$ is the effective water saturation, $\theta_{s}$ is the water content at saturation, which equals porosity $\phi, \theta_{r}$ is the residual water content. The parameter $\alpha_{v}\left(\mathrm{~m}^{-1}\right)$ relates to the air entry pressure, and $n$ and $m$ are two empirical coefficients. 
Substituting eq. 19 in the capillary model from Mualem [1976] yields to the following relative permeability model,

$$
K\left(S_{e}\right)=K_{s} S_{e}^{L}\left[1-\left(1-S_{e}^{1 / m}\right)^{m}\right]^{2}
$$

with $K_{s}$ the hydraulic conductivity at saturation $(\mathrm{m} / \mathrm{s})$. The values of $m$ and $n$ are linked by: $m=1-1 / n$. Usually, this decoupled model is used to improve the results of the parameter optimization of the inverse problem [van Genuchten and Nielsen, 1985]. The parameters used in Linde et al. [2007] are reported in Table 3. Associating $\alpha_{v}$ to $m$ and $n$ values in eq. 19 yields to a large air entry pressure. The resulting capillary fringe is almost $40 \mathrm{~cm}$ (Fig.6). The capillary fringe is a zone in which water pressure heads can be negative, but which remains saturated (Bear 1972).

Allègre et al. [2010] and Allègre et al. [2012] used the model of Brooks and Corey [1964] to address the retention behaviour of the medium as,

$$
S e=\frac{\theta-\theta_{r}}{\theta_{s}-\theta_{r}}=\left\{\begin{array}{l}
\left(\frac{h_{a}}{|h|}\right)^{\lambda}, \text { if } \quad \frac{h_{a}}{|h|}<1 \\
1, \text { if } \quad \frac{h_{a}}{|h|}>1
\end{array}\right.
$$

The parameter $\lambda$ is an estimate of the pore size distribution, characterizing the medium granulometry, the larger the value of $\lambda$ the more homogeneous the medium. The second hydrodynamic parameter $h_{a}$ is the air entry pressure [Brooks and Corey, 1964] which can be related to $\alpha_{v}$ in eq. (19). The relative permeability behaviour followed the law of Mualem [1976] which leads to,

$$
K\left(S_{e}\right)=K_{s} \cdot S_{e}^{L+2+\frac{2}{\lambda}}
$$

The parameters used by Allègre et al. [2010] and Allègre et al. [2012] are reported in Table 3.

The hydrodynamic measurements from Linde et al. [2007] and Allègre et al. [2010] were modelled using the code proposed by Allègre et al. [2012], that solves the electric and hydrodynamic forward problems simultaneously in one dimension. Hydrodynamic behaviour from Mboh et al. [2012] and Allègre et al. [2014] were modelled using Hydrus-1D (www.pc-progress.com), in both cases using the eqs. (19) and (20) for retention and permeability models. The parameter values from the two studies are reported in Table 3.

The figures 6a-h show the measured and simulated pressure heads for the experiments I, II and III, as well as the respective vertical profile of saturation inferred from the modelling results. Additional modelling 
feature include the cumulative outflow measured by Linde et al. [2007], which is a good proxy to check the robustness of the modelling a posteriori.

Even though the experimental conditions were similar for all experiments, one can observe that the dynamics varies a lot from one to another. While 10 hours were necessary for experiment I to stop, it took approximately 14 hours for experiment III, and about 200 hours for experiment II. The maximum water flow was therefore much smaller during experiment II.

The investigated range of saturation is quite similar as far as the entire column is concerned. However, Allègre et al. [2010] and Mboh et al. [2012] had electrodes located in the part of the medium where the saturation significantly decreased (fig. 6c,i) whereas the saturation investigated by Linde et al. [2007] SP measurements ranged between 0.85 and 1 (Fig. 6f).

\subsection{Streaming potential measurements}

During each experiment, streaming potentials were measured between one electrode and a reference located within the saturated part of the column. Additional SP differences can be inferred at any other electrode locations by subtraction of the raw measurements. The figure 7 compiles the raw SP measurements from experiments I and II. Mboh et al. [2012] corrected raw SP signals using a linear relation designed to address a linear drift between the beginning and the end of the experiment. Following a basic procedure widely reported in the literature [e.g., Allègre et al., 2010; Jougnot and Linde, 2013], we started our analysis back from the raw data and shifted them to zero at the experiment start time (Fig. 7a). Shifting the signals to zero assesses that SP do not exist until water is in motion. Mboh et al. [2012] SP correction also intended to account for non-zero electric potential differences at the end of their experiment (i.e., no flow conditions).

Jougnot and Linde [2013] analysed Mboh et al. [2012] dataset in combination with one dataset of their own which was acquired during drainage and imbibition experiments. They argued that electrode drifting can be responsible for non-related SP signals, such as non-zero potential differences. Zhang et al. [2014] showed with numerical simulations that non-zero SP could be observed in no flow conditions without the need for the electrodes to be responsible for it. We later argue that the behaviour of water/air interfaces appearing when 
the medium is unsaturated can be in fact responsible for a priori non-related SP signals. Jougnot and Linde [2013] also wrote that electrode effects were responsible for the behaviour of SP signals observed by Allègre et al. [2010]. Allègre et al. [2015] and Fiorentino et al. [2016] argued that water/air interfaces could be a plausible cause for that behaviour. The importance of water/air interfaces is further developed in section 4. Since Jougnot and Linde [2013] demonstrated that the electrodes they used could be unstable over the course of their experiment, we decided not to include their data in this section.

SP differences for electrode couples were inferred from raw measurements by subtraction of the raw signals (Fig. 7b-c). The experiment III shows consistently decreasing SP as water saturation decreases (Figures 7c), until the dipoles stabilized. The behaviour of SP signals during experiment II is more chaotic with electrodes dipoles showing both decreasing and increasing values when saturation decreases (Fig. 7b)

Experiments II and III experienced very different flow dynamics. The figure 8a-b shows the total pressure differences computed at the location of SP elecrode couples. The recovery of total pressure differences (i.e., pressures differences coming back to equilibrium) which drives the flow, was quite fast in the case of experiment III (Fig.8a). Such a recovery was not reached after 150 hours in the case of experiment II (Fig.8b). The impact of flow dynamics was argued to be of significant effect on the resulting SP response [Allègre et al., 2015].

\subsection{Streaming-potential modelling}

The numerical approach of Allègre et al. [2012] allowed us to model the SP responses of experiments I and III. A constant SPC, equal to $C_{\text {sat }}$, and the model from Guichet et al. [2003] (eq. 17) were tested in the procedure. For both studies, the original modelling was performed using the SPC model from Revil et al. [2007]. For experiment I, the hydrodynamic modelling showed that the SP signals were measured within the part of the column that remained saturated during the experiment (Fig. 6f). The modelling results consequently show that the SP signals can be fairly estimated assuming a constant SPC, that equals $C_{\text {sat }}$ (Fig. 9). Using the SPC model from Guichet et al. [2003] also leads to a good estimation of SP values. The influence of the SPC model is limited in that case since the saturation remains in the range $[0.85 ; 1]$, and the electrical potential is less sensitive to saturation variations. The small SP amplitudes observed by Linde et al. [2007] $(|\Delta V|$ 
$<1.1 \mathrm{mV}$ ) probably result from the small value of the saturated SPC, $C_{s a t}=-7.9 \times 10^{-7} \mathrm{~V}^{\mathrm{Pa}}{ }^{-1}$. The SP signals measured in the transition zone, where $0.85<S_{w}<1$ (at 25, 35, $60 \mathrm{~cm}$ ), exhibit a similar behaviour than the other dipoles located in the saturated part. The experiment I does not show a significant sensitivity of SP signals to water content variations. Additionally, several models including a constant SPC can fairly predict the measurements.

The same approach was used for experiments III (Fig. 10) and experiment IV (Fig. 11). The data from experiment III are not corrected as mentioned in previous section. As for experiment I, the SP are modelled following the approach from Allègre et al. [2012] associated with a constant SPC and Guichet et al. [2003] SPC model. If the trend of SP signals is pretty well described in both cases, the amplitudes of the dipoles from $73 \mathrm{~cm}$ to $109 \mathrm{~cm}$ are underestimated (Fig. 10). The dipoles from 73 to $109 \mathrm{~cm}$ correspond to water saturation ranging from 0.2 to 0.3 . The SP measurements from Mboh et al. [2012] are therefore much more sensitive to water saturation than those from experiment I.

The experimental SPC curves from experiment III are compared to those from Allègre et al. [2010] experiment in figure 12. The shape of the SPC from experiment III is significantly altered if no SP correction is applied (Fig. 12a-c). One can notice the significant scattering when the water saturation goes very low, and increasing SPC, which is due to too small pressure difference when using eq. (9). The relative SPC curves from experiment III now exhibit value larger than 1, which is a feature shared with Allègre et al. [2010] results. Although the relative SPC can show non-monotonous behaviour for $0.6<S_{w}<0.9$ (e.g., $C_{r / 9,8}$ and $C_{r / 7,6}$ in fig. 12c), it generally decreases when saturation decreases. Interestingly, the relative SPC values for experiment II are lower for larger water flow (Fig. 12d), The upper dipole shows higher velocity since the decrease of the water saturation happens for this dipole at the beginning of the drainage. so I would write the reverse which could be be related to the amount and dynamic of water/air interfaces (see next section).

Experiment IV was performed with a shorter column (fig.11e), which explains why the drainage phases lasted less than those of experiment III. A few millivolts SP signals was produced overall, which is similar to experiment III. Figure 11 presents all of six drainage phases. Although phases $d_{1}$ and $d_{2}$ produces a pressure drop and an outflow (Figs. 11a-b), an extremely weak, almost not measurable SP was created. The 
phases $d_{3}$ and $d_{4}$ produced measurable SP drops, that were followed by a recovery phase to zero potential. The following phases are associated to few $\mathrm{mV}$ amplitude SP with no recovery. The beginning of phase $d_{5}$ defines a sort of a water saturation threshold beyond which no recovery occurs anymore. Again, the stabilization of SP signals as soon as the medium is sufficiently unsaturated can easily be related to the influence of water/air interfaces. Allègre et al. [2014] demonstrated that their electrodes were extremely stable during the experiment and not responsible for that stabilization of SP signals. The next section addresses the importance of the water/air interfaces in the SP response for unsaturated conditions.

\section{Importance of the interface water/air}

We saw that the SPC can show a non-monotonous behaviour with the water saturation, and that classical models assuming a monotonous behaviour can not be used for unsaturated conditions. For unsaturated conditions two interfaces have to be taken into account: the interface between the matrix and the water, and the interface between the water and the air. Indeed both interfaces are charged and are described as electric double layer or triple layer, and can contribute to the streaming potential through the electrokinetic coupling. Depending on the quantity, mobility, and evolution of these interfaces, according to different conditions of fluid flow such as drainage or imbibition, these contributions can show different behaviours. We describe below these interfaces, and show some recent results of the modelling of both interfaces using the Lattice Boltzmann approach.

\subsection{Rock/water interface}

The interface between rock and water is related to the existence of an electrical double layer between the rock and the fluid. Minerals forming the rock develop an electric double-layer when in contact with an electrolyte, usually resulting from a negatively charged mineral surface. An electric field is created perpendicular to the surface of the mineral which attracts counterions (usually cations) and repulses anions in the vicinity of the pore matrix interface. The electric double layer (Fig. 13) is made up of the Stern layer, where cations are adsorbed on the surface, and the Gouy diffuse layer, where the number of counterions exceeds the number of anions [Adamson, 1976; Davis et al., 1978; Hunter, 1981]. 
The distribution of the electrical potential $\phi$ within the electrical double layer, perpendicular to the solid surface can be calculated resolving the Poisson's equation:

$$
\nabla^{2} \phi=-\frac{\rho}{\epsilon_{f}}
$$

where $\epsilon_{f}$ is the dielectric constant of the fluid. The fluid contains $M_{i}$ ionic species with valence $z_{i}\left(\mathrm{i}=1, \ldots . . M_{i}\right)$ and number density $N_{i}^{b}$ (the number of species-i ions per unit volume) in the bulk solution far from any charged surface. The charge density $\rho$ can be expressed using a Boltzmann distribution for the ionic species within the fluid:

$$
\rho=\sum_{i=1}^{M} e z_{i} N_{i}^{b} \exp \left(-\frac{e z_{i} \phi}{k T}\right)
$$

where $\mathrm{k}$ is the Boltzmann constant, -e is the charge of an electron, $\mathrm{T}$ is the temperature. It is often assumed that the Poisson-Boltzmann equation governing the equilibrium charge clouds can be linearized. Assuming $e \phi / k T<<1$, the Poisson's equation becomes:

$$
\nabla^{2} \phi=\left(d^{l}\right)^{-2} \phi
$$

with $d^{l}$ the Debye length, which is a measure of the thickness of the double diffuse layer, typically of the order of a few nm:

$$
\frac{1}{\left(d^{l}\right)^{2}}=\sum_{i=1}^{M} \frac{e^{2} z_{i}^{2} N_{i}^{b}}{\epsilon_{f} k T}
$$

The electrical potential $\phi$ at a distance $x$ from a charged surface is therefore

$$
\phi(x)=\zeta \exp \left(-\frac{x}{d^{l}}\right)
$$

and $\zeta$ is called the zeta potential and is the electrical potential at the shear plane (for further details see Pride [1994]). The zeta potential itself depends on rock, fluid composition, and pH [Ishido and Mizutani, 1981; Jouniaux et al., 1994, 2000; Jouniaux and Pozzi, 1995; Lorne et al., 1999; Guichet et al., 2006; Maineult et al., 2006b; Jaafar et al., 2009; Vinogradov et al., 2010].

The charge density at the surface of the minerals results from surface complexation reactions. The quartz surface can be modelled with silanol $>$ SiOH group [Davis et al., 1978]. The potential-determining ions 
$\mathrm{OH}^{-}$and $\mathrm{H}^{+}$are adsorbed onto the surface of the mineral and determine the charge density on the inner plane (see figure 13). Therefore the surface charge depends on the $\mathrm{pH}$. There exists a $p H$ for which the total surface charge is zero: this is the point of zero charge and $p H$ is called $p H_{p z c}$ [Davis and Kent, 1990; Sposito, 1989]. The charge is positive for $p H<p H_{p z c}$ and negative for $p H>p H_{p z c}$. In this case this electrokinetic effect is zero. The $p H_{p z c}$ for quartz is in the range $2<p H_{p z c}<4$ [Parks, 1965; Lorne et al., 1999]. The calcite surface can be modelled with $>\mathrm{CaOH}$ and $>\mathrm{CO}_{3} \mathrm{H}$ groups. Carbonate ions and $\mathrm{Ca}^{2+}$ are the determining-potential ions. The electrokinetic behavior on carbonates is more complicated. The $p H_{p z c}$ varies from 7 to 10.8 according to the authors [VanCappellen et al., 1993].

It is possible to model simple interfaces and to calculate zeta potential in simple cases [Guichet et al., 2006]. This modeling can be performed assuming the triple-layer-model (TLM) which distinguishes three planes to describe the electric double layer: the inner Helmholtz plane for counter ions directly bound to the mineral (assumed to be chemically adsorbed), the outer Helmholtz plane for weakly bound counter ions (assumed to be physically adsorbed), and a $d$-plane associated with the smallest distance between the mineral surface and the counter ions in the diffuse layer. It has been proposed that the slipping plane lies near the distance of closest approach of dissociated ions and that the $\zeta$ potential can be calculated as the potential on this plane [Davis and Kent, 1990].

At a given $\mathrm{pH}$ the most influencing parameter on the streaming potential coefficient is the fluid conductivity. It has been proposed that $C_{0}=-1.2 \times 10^{-8}\left(\sigma^{f}\right)^{-1}$ [Allègre et al., 2010], based on data collected in the literature on sandstones and sands (Fig. 14), which leads to a zeta potential equal to $-17 \mathrm{mV}$ assuming Eq. (9) and that zeta potential, dielectric constant, and viscosity do not depend on fluid conductivity. These assumptions are not exact, but the value of zeta is needed for numerous modellings which usually assume the dielectric constant and viscosity independent of the fluid conductivity. Luong and Sprik [2014] also proposed that the zeta potential is constant over a large range of electrolyte concentration. A recent study, taking into account the viscosity and permittivity relationship with the electrolyte concentration, deduced a constant apparent zeta potential of $-21 \mathrm{mV}$, from the comparison of modelling and experimental data set from the literature [Fiorentino et al., 2016]. Therefore an average value of an apparent zeta $-20 \mathrm{mV}$ for the needed input value in modellings is fairly exact, at least for media with no clay nor calcite, and in the fluid conduc- 
tivity range excepting very high values. Another formula is often used [Pride and Morgan, 1991] based on quartz minerals rather than on sands and sandstones, which may be less appropriate for field applications when this formula is used in the models without taking into account the effect of the fluid conductivity on all the parameters leading to biaises values of the streaming potential coefficients..

The streaming current is due to the motion of the diffuse layer induced by a fluid pressure difference along the interface. It has been proposed that the streaming current could be used to deduce the permeability [Jouniaux, 2011; Wang et al., 2016]. This streaming current is then balanced by the conduction current, leading to the streaming potential which is proportional to the zeta potential (eq.9): the knowledge of the electric double layer of the rock/water interface and its zeta potential is therefore essential. In the same way the knowledge of the water/air interface is essential and is described below.

\subsection{Water/air interface}

Recently Allègre et al. [2015] showed that the interface between water and air should also be taken into account, since this interface is negatively charged, as the interface between the rock matrix and the water.

The interface developed between water and air is negatively charged (Fig. 15), the associated zeta potential being negative and ranging from $-65 \mathrm{mV}$ to $-35 \mathrm{mV}$ in distilled water [e.g., Graciaa et al., 1995; Takahashi, 2005]. For a water electrical conductivity of $10^{-2} \mathrm{~S} / \mathrm{m}$ (equivalent to $\mathrm{NaCl}$ concentration of $10^{-3}$ $\mathrm{mol} / \mathrm{l}$ ) and for $\mathrm{pH}=7$, the zeta potential ranges from $-30 \mathrm{mV}$ to $-40 \mathrm{mV}$ [e.g., Yang et al., 2001; Creux et al., 2007]. We can note that sand/water interfaces are also negatively charged, the associated zeta potential being equal to $-20 \mathrm{mV}$. When relative motion occurs at the shear plane of the air/water interface, this results in a streaming current density identical in sign to the current classically created at water/grain interfaces. On the contrary, if the water/air interfaces are dragged, and there is no relative motion, no additional streaming current will occur.

\subsection{Evolution of the water/air interface during a drainage}

One should consider that the streaming current depends on the amount of charges mobilized during a flow. The excess countercharge density depends on the electrical characteristics of the rock/water interface 
and it has been shown that the matrix/water interfacial area decreases monotonously with decreasing watersaturation (Fig. 16) [Culligan et al., 2006].

However the excess countercharge density should also depend on the water/air interfaces in the case of multi-phase flow (water/air/rock). During a drainage phase the surface-area of the water/air interfaces does not decrease with decreasing water-saturation, but first rises to a maximum, and finally decreases. This behaviour has been observed during drainage experiments (Fig. 17) [Culligan et al., 2004], and has been also modelled [e.g., Reeves and Celia, 1996; Berkowitz and Hansen, 2001]).

We argue that the behaviour of the unsaturated SPC is related to the surface-area of the water/air interfaces, since it displays a non-monotonous behaviour as a function of water saturation. This quantity affects the cohesion of the material: the larger the surface-area of the interfaces, the larger the cohesive stress of the material. It has been shown that the cohesive stress first increases with decreasing water saturation (capillary regime), then reaches a maximum value (within the funicular regime), and finally decreases with further decreasing saturation [e.g., Mitarai and Nori, 2006] (see Fig. 18). During a drainage experiment of a sand column, the upper dipoles first undergo a capillary phase followed by a funicular phase. The uppermost sand may be subject to a pendular regime.

However to quantify the relative importance of the contribution of the surface-area of the water/air interface to the surface-area of the matrix/water interface, we developed a two-phase lattice Boltzmann modelling, whose first results are described in the following section.

\subsection{Two-phase modelling of steaming potential}

The streaming potential coefficient has been modelled solving the Navier-Stokes equation and the PoissonBoltzmann equation, using the lattice Boltzmann method in a 2D capillary channel [Fiorentino et al., 2016]. This method allows to model the electrokinetic coupling at a small scale. The flow of an electrolyte within a channel is simulated, as well as the accumulation of charges at the rock/water and water/air interfaces. Then these results are coupled through the computation of the electric field induced by the water flow, via a second Lattice Boltzmann model solving the Poisson-Boltzmann equation. The macroscopic streaming poten- 
tial coefficient is deduced from averaging the electric field on the entire channel and from the pressure difference allowing the fluid flow. The presence of water and air within the channel has been studied through several cases: a- the water and the air flows are parallel; b- the air is entrapped within the pores; c- the air flows through bubbles [Fiorentino et al., 2017]. The zeta potential of both interfaces is set at $-20 \mathrm{mV}$.

\subsubsection{Air flow parallel to the channel}

The water content is decreased by increasing the air content in the middle of the channel: the interface water/air is therefore parallel to the interface matrix/water, and the diameter of this air corridor is increasing while the water saturation is decreasing. In this case the surface area of the water/air interface is continuous and constant whatever is the water saturation, except at full water saturation. The streaming potential coefficient is calculated as a function of the water saturation: $C_{E K}^{a l l}$ involves all the sites of the channel, $C_{E K}^{w}$ involves the sites within the water excluding the sites within the air which is not a conducting phase.

The streaming potential coefficient is increasing with increasing water saturation, up to the full water saturation for which the interface water/air does not exist anymore, leading to a decrease of the streaming potential coefficient (Fig.19). These results show a non monotonous behaviour of the streaming potential coefficient with the water saturation, without involving an increase of the surface area of the water/air interface. The increase of the SPC with increasing saturation is linked to the increase of the fluid velocity at the interface water/air which is moving towards the centre of the channel when the water saturation is increasing. Therefore the positive charge excess of the water/air interface is enhanced by a larger velocity, leading to an increase of the SPC.

\subsubsection{Flow with entrapped bubbles}

Air bubbles are entrapped on the wall of the channel, with an increasing size for a decreasing water saturation. The resulting SPC is also non monotonous with the water saturation with a possible increase within the range $95 \%$ to $80 \%$ water saturation (Fig.20). In this case the fluid velocity is decreasing with decreasing water saturation, and the charge density and the surface area of the water/air interface is increasing, resulting in such a behaviour of the SPC. 
Air injection in the channel is simulated by flowing an increasing number of bubbles in the centre of the channel, that finally merge together. The streaming potential coefficient shows a non monotonous behaviour with water saturation, linked to a decrease of the fluid velocity with decreasing water saturation, and to an increasing charge density and surface area of the water/air interface with decreasing water saturation (Fig.21). The step in SPC between $55 \%$ and $50 \%$ is linked to the fact that two bubbles merge into one bubble. The value of the SPC at $82 \%$ (star) corresponds to a configuration with more than two bubbles and shows a higher value compared to the SPC value at $82 \%$ with two bubbles. The SPC values (dots) involve a zeta potential of $-20 \mathrm{mV}$ for both matrix/water and water/air interface, whereas the SPC values (crosses) are calculated with a null zeta potential at the interface water/air. The results are therefore very different when the interface water/air is not taken into account. The non monotonous behaviour of the SPC can hardly be seen when the water/air interface is neglected: the main contribution to the SPC comes from the water/air interface.

Therefore these numerical results show that the water/air interface is the main contribution in the electrokinetic signal for unsaturated conditions, even if the surface area of this interface is constant. An increase of this interface further contribute to the electrokinetic signal. It has to be noted that we made the assumption that the negative charges causing the zeta potential at the interface air/water are placed in the air phase and stay in the air. They do not contribute to the calculation of the SPC. If these negative charges were in the water, they would contribute to the local electric charge density. In the case of flowing bubbles these charges would move and induce an electric current balancing the electric current due to the excess positive charges. Then the enhancement of the SPC with decreasing saturation would be linked to the difference of velocity between air and water which is near zero. Therefore for flowing bubbles the SPC would not be increased with decreasing saturation, whereas for fixed bubbled the SPC would still first increase with decreasing saturation and then decrease with further decreasing saturation. We discuss in the following section the effect of different behaviour of the streaming potential coefficient on seismoelectric conversions for unsaturated conditions. 


\section{Consequences on seismoelectric conversions for nonsaturated cases}

We now describe how the behaviour of the streaming potential coefficient for unsaturated conditions is taken into account in the modelling of the seismoelectric conversions. Then we summarize the seismoelectric observations for unsaturated conditions.

\subsection{Seismoelectric modelling for unsaturated conditions}

An extension of Pride's equations aiming to take into account unsaturated materials, in the case of a water-air mixture has been developed by Warden et al. [2013]. The effective medium theory is used to express mechanical properties such as the bulk and shear moduli and dynamic viscosity (see Table 2). The medium's permittivity is derived using the Complex Refractive Index Method, while its conductivity is obtained by extending the conductivity derived by Pride [1994] (Equation 242) to partial saturation conditions; this expression takes the surface conductivities into account. This approach is combined with the strategy introduced by Strahser et al. [2011], thus writing the dynamic seismoelectric coupling under partial saturation conditions as a function of the saturation-dependent Streaming Potential Coefficient (SPC).

In order to express the static seismoelectric coupling $\mathcal{L}_{0}$ under partial saturation conditions, the SPC at full saturation in Equation 7 is replaced by the saturation-dependent SPC given in Equation 12. One also needs to replace the rock effective conductivity at full saturation in Equation 7 with a saturation-dependent effective conductivity. Using Archie's second law, that is, neglecting surface conductivities:

$$
\sigma=\frac{\sigma^{f}}{F} S_{w}^{n}=\sigma^{f} \phi^{m} S_{w}^{n}=\sigma^{f} \frac{\phi}{\alpha_{\infty}} S_{w}^{n}
$$

Therefore, $\mathcal{L}_{0}\left(S_{w}\right)$ can be written as:

$$
\mathcal{L}_{0}\left(S_{w}\right)=-\frac{1}{F} \frac{\epsilon_{f} \zeta}{\eta}\left(1-2 \frac{\tilde{d}}{\Lambda}\right) S_{w}^{n} S\left(S_{w}\right)
$$

with $S_{w}$ a function of saturation being possibly different (see Table 1). 
By implementing this approach in the GMRT method by Garambois and Dietrich [2002], Warden et al. [2013] studied the interface responses (IR) created by a saturation contrast, and evaluated the influence of the presence of a capillary fringe. They concluded that an IR created by a saturation contrast between sand and sandstone may be easier to detect than a seismoelectric conversion occurring at the same boundary between sand and sandstone with the two units fully saturated. Moreover, they proved that the conversions depend on the type of saturation transition existing between the partially saturated and fully saturated units, as it is depicted in Fig. 22. The extension for the electrokinetic coefficient using different $S\left(S_{w}\right)$ to handle partially saturated media was later used to perform computational simulations of the interface responses at different saturations in a $\mathrm{CO}_{2}$ geological deposition site [Zyserman et al., 2015], and to analyze the contamination of a fresh water aquifer by dense non-aqueous liquids [Munch and Zyserman, 2016]. In the former, it was observed that the IR are sensitive to $\mathrm{CO}_{2}$ saturations ranging between $10 \%$ and $90 \%$, and that the $\mathrm{CO}_{2}$ saturation at which the IR maxima are reached depends on the models employed for $S\left(S_{w}\right)$. Moreover, the simulations predict that the IR's are still sensitive to different $\mathrm{CO}_{2}$ saturations for a sealed $\mathrm{CO}_{2}$ reservoir covered by a clay layer. For the latter, it was observed that the predicted IR's are sensitive to different contaminants saturation only when the thickness of the contaminated layer is larger than a threshold value depending on the properties of the non-aqueous fluid and the saturation function $S\left(S_{w}\right)$; in Fig. 23 this situation is depicted.

Other authors, e.g., Jardani et al. [2010]; Revil and Jardani [2010]; Revil and Mahardika [2013] proposed a model alternative to Eq.(29) for the electrokinetic coupling at partial saturation; instead of working with the zeta potential, they based the coupling on the excess of electrical charge present in the pore water. However, their model also employs a still not validated -to the authors' knowledge- relationship between the mentioned excess charges and the rock permeability; this point is discussed by Jouniaux and Zyserman [2015], so that this approach should not be used unless the relation between the excess charge density and the permeability is proved. Within this frame, Revil and Mahardika [2013] studied two-phase flow conditions and proposed a numerical application for water flooding of a non-aqueous phase liquid (NAPL, oil) contaminated aquifer where they observed that seismoelectric conversions mostly take place at the NAPL (oil)/water invasion front and could be used to remotely track its position; Sava et al. [2014] proposed a method to im- 
age the soil electrical resistivity between a set of wells, by mapping the interfaces between different formations by means of seismoelectrics, and afterwards deriving the electrical resistivity using crosswell electric tomography. They concluded that the proposed methodology could resolve the fine structure of resistivity between the wells.

\subsection{Seismoelectric observations for unsaturated conditions}

The effect of moisture on seismoelectrics was studied in laboratory through experiments performed on samples. However it is difficult to deduce a behaviour of the transfer function as a function of the water-saturation, because of different conditions in the laboratory experiments.

The seismoelectric (SE) potential was measured to increase with increasing moisture from 1 to $17 \%$ on samples of limestone, sandstone, aleurolites and marl. At moisture in excess of $15 \%$ a slight decrease was observed in some samples. The inflection of this curve was shifted toward higher moisture values in proportion to the increase in the concentration of the solution [Gaskarov and Parkhomenko, 1974]. A sharp increase at low water content was observed in other studies, and could then be constant at increasing water content on dolomite, marl and sandstones, or could decrease on tegillate loam, morainic loam, and limestones for a frequency of the seismic source around $25 \mathrm{kHz}$ [Parkhomenko and Tsze-San, 1964; Parkhomenko and Gaskarov, 1971; Ageeva et al., 1999]. However, at low frequencies (400 Hz compared to $25 \mathrm{kHz}$ ) no decrease of the SE effect was observed with increasing water saturation. Only Ageeva et al. [1999] performed measurements at low frequencies $(400 \mathrm{~Hz})$, but they normalized the SE signal to the response of the source of the elastic waves (the test transducer, in V), so that the coseismic transfer function (Eq. 30) cannot be deduced.

Seismo-electric conversions in the field can be observed at different conditions of water-content. The water content of the soil can be deduced from electrical resistivity survey or georadar survey. Strahser et al. [2011] observed seismo-electric conversions in the field, as a function of water-saturation, and proposed a transfer function between the electric field and the acceleration as a function of the water-saturation. These authors proposed to modify the transfer function developed by Garambois and Dietrich [2001], in the low frequency assumption valid at seismic frequencies, meaning at frequencies lower than the Biot's frequency sep- 
arating viscous and inertial flows. In this case, and assuming the Biot's moduli $C \ll H$, Garambois and

Dietrich [2001] showed that the SE field $\mathbf{E}$ is proportional to the grain acceleration for longitudinal fast $P$ waves:

$$
\mathbf{E} \simeq-\frac{\mathcal{L}_{0}}{\sigma} \rho^{\mathrm{f}} \ddot{\boldsymbol{u}}=\frac{\epsilon_{\mathrm{f}} \zeta}{\eta \sigma^{\mathrm{f}}} \rho^{\mathrm{f}} \ddot{\boldsymbol{u}} .
$$

Based on their field observations Strahser et al. [2011] proposed that in the low frequency domain, taking into account the water saturation, the SE field and the seismic field are related as:

$$
\mathbf{E} \simeq \frac{\epsilon \zeta}{\eta \sigma^{\mathrm{f}}} S_{\mathrm{e}}^{(0.42 \pm 0.25) n} d_{\mathrm{f}} \ddot{\boldsymbol{u}} .
$$

These observations could not be performed in a large range of water-content, leading to relatively scattered data.

Recently the effect of water saturation on coseismic SE signals was studied on sand [Bordes et al., 2015]. Experiments were performed during imbibition and drainage for several cycles, and the water-content was measured by capacitance probes. These authors developed as seismic source a steel ball hitting a granite cylinder in contact with the sand [Sénéchal et al., 2010], showing a main frequency content of about $1.5 \mathrm{kHz}$ and inducing direct $P$ wave [Barrière et al., 2012]. The electric signal was recorded by electrodes dipoles (10 $\mathrm{cm}$ apart) along the $P$ wave propagation. The authors estimated the transfer function of the electric field (electric field over acceleration) by picking the arrival in time domain, and by a spectral analysis using continuous wavelet transform. Both methods showed that these ratios are of the order of $2-7 \times 10^{-4} \mathrm{~V} \mathrm{~m}^{-2} \mathrm{~s}^{-2}$ (depending on the offset to the source) and are rather constant in the water saturation range 0.2-0.9 for imbibition and drainage experiments. None of the tested models for the water-saturation dependence of the SPC could model correctly a constant transfer function in this range of saturation.

\section{Discussion and conclusions}

Measurements of SP for unsaturated conditions published in the literature do not always show the same trend, because they can depend on the conditions of the experiment. Whether the experiment consists of a drainage phase, of an imbibition phase, or uses air injection, results in very different pressure behaviours (Fig. 8), and leads to a different SP response. We show that the raw SP data should not be corrected for a drift if the electrodes are stable in a saturated medium. The apparent drift of the SP signals is due to water/air 
interfaces, which are negatively charged such as rock/water interfaces. Such a drift is therefore part of the useful signal.

Relevant unsaturated SPC curves can be inferred from raw observations if the experiments investigate a large saturation range. We showed that the model tested in Linde et al. [2007] is based on SP measurements performed at saturation ranging from 0.85 to 1 , and that these signals [Linde et al., 2007] can be fairly well simulated using a constant SPC equal to $C_{s a t}$ (Fig. 9). Therefore, one should not rely on this study to interpret SP measurements out of that saturation range. The SPC models from Revil et al. [2007] and Linde et al. [2007] models, assuming that the excess of charge in the diffuse layer to saturation $\left(Q_{v, \text { sat }}\right)$ scales $1 / S_{w}$, is not supported by these observations if $S_{w}<0.85$.

The underlying physics of a non-monotonous behaviour of the SPC is related to water/air interfaces as shown by the results of the lattice Boltzmann numerical simulations. Whether the water/air interfaces progresses as an air flow parallel to the channel, or as a flow with entrapped bubbles, or as flowing bubbles, the results predict a non-monotonous behaviour of the SPC with the water saturation. The main contribution to the SPC comes from the charged water/air interface, and not from the rock/water interface, assuming the same zeta potential for both interfaces. Two competing effects are involved: the decrease of the fluid velocity with decreasing water saturation, and the increase of the charge density and the surface area of the water/air interface with decreasing water saturation. A non monotonous behaviour of the SPC also appears without any increase of the surface area of the water/air interface with decreasing water saturation.

One should use a non-monotonous SPC in order to model seismoelectric conversions. This assumption allows a larger SPC value for saturation ranging from 0.6 to 1 leading to larger amplitudes of seismolectric conversions However, seismoelectric conversions occurring for unsaturated conditions do not only rely on the saturation dependence of the SPC [Bordes et al., 2015; Warden et al., 2013], and observations and modelling are still not always coherent. 


\section{Acknowledgements}

We thank INSU-CNRS and the University of Strasbourg for their support. FZ acknowledges partial support from CONICET through grant PIP 112-201501-00192.

\section{References}

Adamson, A. W. (1976), Physical chemistry of surfaces, 698 pp., John Wiley and sons, New York.

Ageeva, O. A., B. S. Svetov, G. K. Sherman, and V. Shipulin (1999), E-effect in rocks, Russian Geology and Geophysics, 64, 1349-1356.

Ahmad, M. (1964), A laboratory study of streaming potentials, Geophys. Prospect., XII, 49-64.

Aizawa, K., M. Uyeshima, and K. Nogami (2008), Zeta potential estimation of volcanic rocks on 11 island arc-type volcanoes in japan: implication for the generation of local self-potential anomalies, $J$. Geophys. Res., 113, B02,201.

Alkafeef, S., and A. Alajmi (2006), Streaming potentials and conductivities of reservoir rock cores in aqueous and non-aqueous liquids, Colloids and Surfaces A: Physicochem Eng. Aspects, 289, 141-148, doi:10.1016/j.colsurfa.2006.04.023.

Allègre, V., L. Jouniaux, F. Lehmann, and P. Sailhac (2010), Streaming Potential dependence on water-content in fontainebleau sand, Geophys. J. Int., 182, 1248-1266, doi:10.1111/j.1365246X.2010.04716.x.

Allègre, V., L. Jouniaux, F. Lehmann, and P. Sailhac (2011), Reply to the comment by A. Revil and N. Linde on: "Streaming potential dependence on water-content in fontainebleau sand" by V. Allègre, L. Jouniaux, F. Lehmann and P. Sailhac, Geophys. J. Int., 186, 115-117.

Allègre, V., F. Lehmann, P. Ackerer, L. Jouniaux, and P. Sailhac (2012), Modelling the streaming potential dependence on water content during drainage: 1. A 1D modelling of SP using finite element method, Geophys. J. Int., 189, 285-295, doi:10.1111/j.1365-246X.2012.05371.x.

Allègre, V., A. Maineult, F. Lehmann, F. Lopes, and M. Zamora (2014), Self-potential response to drainage-imbibition cycles, Geophys. J. Int., 197, 1410-1424, doi:10.1093/gji/ggu055. 
Allègre, V., L. Jouniaux, F. Lehmann, P. Sailhac, and R. Toussaint (2015), Influence of water pressure dynamics and fluid flow on the streaming-potential response for unsaturated conditions, Geophysical Prospecting, 63, 694-712, doi:10.1111/1365-2478.12206.

Archie, G. E. (1942), The electrical resistivity log as an aid in determining some reservoir characteristics, Trans. Am. Inst. Min. Metall. Pet. Eng., (146), 54-62.

Barrière, J., C. Bordes, D. Brito, P. Sénéchal, and H. Perroud (2012), Laboratory monitoring of p waves in partially saturated sand, Geophys. J. Int., 191(3), 11521170.

Bekri, S., J. Howard, J. Muller, and P. Adler (2003), Electrical resistivity index in multiphase flow through porous media, Transport in Porous media, 51, 41-65.

Berkowitz, B., and D. Hansen (2001), A numerical study of the distribution of water in partially saturated porous rock, Transport in Porous Media, 45, 303-319.

Block, G. I., and J. G. Harris (2006), Conductivity dependence of seismoelectric wave phenomena in fluid-saturated sediments, J. Geophys. Res., 111, B01,304, doi:10.1029/2005JB003798.

Bordes, C., P. Sénéchal, J. Barrière, D. Brito, E. Normandin, and D. Jougnot (2015), Impact of water saturation on seismoelectric transfer functions:a laboratory study of coseismic phenomenon, Geophys. J. Int., 200(3), 1317-1335, doi:10.1093/gji/ggu464.

Brie, A., F. Pampuri, A. Marsala, and O. Meazza (1995), Shear sonic interpretation in Gas Bearing sands, Society of Petroleum Engineers.

Brooks, R. J., and A. T. Corey (1964), Hydraulic properties of porous media, Hydrol. Pap., 3, 318-333.

Brothelande, E., A. Finizola, A. Peltier, E. Delcher, J.-C. Komorowski, F. D. Gangi, G. Borgogno, M. Passarella, C. Trovato, and Y. Legendre (2014), Fluid circulation pattern inside la soufrire volcano (guadeloupe) inferred from combined electrical resistivity tomography, self-potential, soil temperature and diffuse degassing measurements, J. Volc. Geotherm. Res., 288, 105-122, doi: 10.1016/j.jvolgeores.2014.10.007.

Carrara, E., A. Mazzaca, R. Pece, N. Roberti, and T. Vanorio (1999), Evaluation of porosity and saturation degree by laboratory joint measurement of velocity and resistivity: a model improvement, Pure Appl. Geophys., (154), 211-255. 
Chandler, R. (1981), Transient streaming potential measurements on fluid-saturated porous structures:

An experimental verification of Biot's slow wave in the quasi-static limit, J. Acoust. Soc. Am., 70, $116-121$.

Cooke, C. E. (1955), Study of electrokinetic effects using sinusoidal pressure and voltage, J. Chem. Phys., (23), 2299-2303.

Creux, P., J. Lachaise, A. Graciaa, and J. Beattle (2007), Specific cation effects at the hydroxide-charged air/water interface, J. Phys. Chem. C, 111, 3753-3755, doi:10.1021/jp070060s.

Culligan, K., D.Wildenschild, B. Christensen, W.G.Gray, M. Rivers, and A. Tompson (2004), Interfacial area measurements for unsaturated flow through a porous medium, Water Resources Reasearch, 40, W12,413, doi:10.1029/2004WR003278.

Culligan, K., D. Wildenschild, B. Christensen, W. Gray, and M. Rivers (2006), Pore-scale characteristics of multiphase flow in porous media: A comparison of air-water and oil-water experiments, Advances in Water Resources, 29(1), 227-238, doi:10.1016/j.advwatres.2005.03.021.

Davis, J., and D. Kent (1990), Surface complexation modeling in aqueous geochemistry, in Mineral Water Interface Geochemistry, M.F. Hochella and A.F. White, Mineralogical Society of America.

Davis, J. A., R. O. James, and J. Leckie (1978), Surface ionization and complexation at the oxide/water interface, J. Colloid Interface Sci., 63, 480-499.

Desroches, A., and K. Butler (2016), Monitoring and modeling of pumping-induced self potentials for transmissivity estimation within a heterogeneous confined aquifer, Geophys. J. Int., First published online: September 22, doi:10.1093/gji/ggw354.

Dukhin, S. S., and B. V. Derjaguin (1974), Surface and Colloid Science, edited by E. Matijevic, John Wiley and sons, New York.

Dupuis, J. C., K. E. Butler, and A. W. Kepic (2007), Seismoelectric imaging of the vadose zone of a sand aquifer, Geophysics, 72, A81-A85, doi:10.1190/1.2773780.

Dupuis, J. C., K. E. Butler, A. W. Kepic, and B. D. Harris (2009), Anatomy of a seismoelectric conversion: Measurements and conceptual modeling in boreholes penetrating a sandy aquifer, J. Geophys. Res. Solid Earth, 114(B13), B10,306, doi:10.1029/2008JB005939. 
Fiorentino, E., R. Toussaint, and L. Jouniaux (2016), Lattice boltzmann modelling of streaming potentials: variations with salinity in monophasic conditions, Geophys. J. Int., 205, 648664, doi: 10.1093/gji/ggw041.

Fiorentino, E., R. Toussaint, and L. Jouniaux (2017), Two-phase lattice boltzmann modelling of streaming potentials: influence of the gas-water interface on the electrokinetic coupling, Geophys. J. Int., 208, 1139-1156, doi:10.1093/gji/ggw417.

Fournier, C. (1989), Spontaneous potentials and resistivity surveys applied to hydrogeology in a volcanic area: case history of the chaine des puys (france), Geophysical Prospecting, 37, 647-668.

Gaffet, S., Y. Guglielmi, J. Virieux, G. Waysand, A. Chwala, R. Stolz, C. Emblanch, M. Auguste, D. Boyer, and A. Cavaillou (2003), Simultaneous seismic and magnetic measurements in the lownoise underground laboratory (lsbb) of rustrel, france, during the 2001 january 26 indian earthquake, Geophys. J. Int., 155, 981-990.

Gao, Y., X. Chen, H. Hu, and J. Zhang (2013), Early electromagnetic waves from earthquake rupturing:ii validation and numerical experiments, Geophys. J. Int., 192(3), 1308-1323, doi: 10.1093/gii/ggs097.

Gao, Y., J. Harris, J. Wen, Y. Huang, C. Twardzik, X. Chen, and H. Hu (2016), Modeling of the coseismic electromagnetic fields observed during the 2004 mw 6.0 parkfield earthquake, Geophys. Res. Lett., $43,620-627$

Garambois, S., and M. Dietrich (2001), Seismoelectric wave conversions in porous media: Field measurements and transfer function analysis, Geophysics, 66, 1417-1430.

Garambois, S., and M. Dietrich (2002), Full waveform numerical simulations of seismoelectromagnetic wave conversions in fluid-saturated stratified porous media, J. Geophys. Res., 107(B7), ESE 5-1, doi:10.1029/2001JB000316.

Gaskarov, I., and E. Parkhomenko (1974), The seismoelectric effect in rocks and the preconditions for its application in geological prospecting work, Izv. Akad. Sci. USSR, Physics Solid Earth, 1, 71-74.

Giampaolo, V., D. Calabrese, and E. Rizzo (2016), Transport processes in porous media by selfpotential method, Applied and Environmental Soil Science, volume2016, Article ID 3951,486, doi: 
Glover, P., and N. Déry (2010), Streaming potential coupling coefficient of quartz glass bead packs: dependence on grain diameter, pore size, and pore throat radius, Geophysics, 75, F225-F241.

Glover, P., and M. Jackson (2010), Borehole electrokinetics, The Leading Edge, pp. 724-728.

Glover, P., E. Walker, J. Ruel, and E. Tardif (2012), Frequency-dependent streaming potential of porous media-Part 2: Experimental mesurement of unconsolidated materials, Int. J. Geophysics, 2012(Hindawi Publishing Corporation), Article ID 728,495, doi:10.1155/2012/728495.

Gonzales, K., A. Finizola, J. Lénat, O. Macedo, D. Ramos, J. Thouret, N. Fournier, V. Cruz, and K. Pistre (2014), Asymmetrical structure, hydrothermal system and edifice stability: The case of ubinas volcano, peru, revealed by geophysical surveys, J. Volc. Geotherm. Res., 276, 132-144, doi: dx.doi.org/10.1016/j.jvolgeores.2014.02.020.

Graciaa, A., G. Morel, P. Saulnier, J. Lachaise, and R. Schechter (1995), J. Colloid Interface Sci., 172, 131.

Groves, J., and A. Sears (1975), Alternating streaming current measurements, J. Colloid Interface Sci., 53, 83-89.

Guan, W., H. Hu, and Z. Wang (2013), Permeability inversion from low-frequency seismoelectric logs in fluid-saturated porous formations, Geophysical Prospecting, 61, 120-133, doi:10.1111/j.13652478.2012.01053.x.

Guglielmi, A., B. Tsegmed, A. Potapov, I. Kultima, and T. Raita (2006), Seismomagnetic signals from the strong earthquake of sumatra, Izvestiya, Physics of the Solid Earth, 42, 921-927.

Guichet, X., L. Jouniaux, and J.-P. Pozzi (2003), Streaming potential of a sand column in partial saturation conditions, J. Geophys. Res., 108(B3), 2141, doi:10.1029/2001JB001517.

Guichet, X., L. Jouniaux, and N. Catel (2006), Modification of streaming potential by precipitation of calcite in a sand-water system: laboratory measurements in the $\mathrm{pH}$ range from 4 to 12 , Geophys. J. Int., 166, 445-460, doi:10.1111/j.1365-246X.2006.02922.x.

Hase, H., T. Ishido, S. Takakura, T. Hashimoto, K. Sato, and Y. Tanaka (2003), Zeta potential measurement of volcanic rocks from aso caldera, Geophys. Res. Lett., 23(30), 2210, doi: 
Hornbostel, S., and A. Thompson (2007), Waveform design for electroseismic exploration, Geophysics, 72(2), Q1-Q10, doi:10.1190/1.2436473.

Hu, H., and J. Liu (2002), Simulation of the converted electric field during acoustoelectric logging, 72nd SEG Annual International Meeting, Expanded Abstracts,21(Salt Lake City, Utah, USA), 348-351, doi:10.1029/2001JB001517.

Hu, H., W. Guan, and J. Harris (2007), Theoretical simulation of electroacoustic borehole logging in a fluid-saturated porous formation, J. Acoust. Soc. Am., 122, 135-145.

Hunt, C. W., and M. H. Worthington (2000), Borehole elektrokinetic responses in fracture dominated hydraulically conductive zones, Geophys. Res. Lett., 27(9), 1315-1318.

Hunter, R. (1981), Zeta Potential in Colloid Science: Principles and Applications, 386 pp., Academic., New York.

Ishido, T., and H. Mizutani (1981), Experimental and theoretical basis of electrokinetic phenomena in rock water systems and its applications to geophysics, J. Geophys. Res., 86, 1763-1775.

Ishido, T., and J. Pritchett (1999), Numerical simulation of electrokinetic potentials associated with subsurface fluid flow, J. Geophys. Res., 104(B7), 15,247-15,259.

Jaafar, M. Z., J. Vinogradov, and M. D. Jackson (2009), Measurement of streaming potential coupling coefficient in sandstones saturated with high salinity nacl brine, Geophys. Res. Lett., 36, L21,306, doi:10.1029/2009GL040549.

Jackson, M. D. (2008), Characterization of multiphase electrokinetic coupling using a bundle of capillary tubes model, J. Geophys. Res., 113, B04,201, doi:10.1029/2007JB005490,2008.

Jackson, M. D. (2010), Multiphase electrokinetic coupling: Insights into the impact of fluid and charge distribution at the pore scale from a bundle of capillary tubes model, J. Geophys. Res., 115, B07,206, doi:10.1029/2009JB007092,2010.

Jardani, A., A. Revil, E. Slob, and W. Söllner (2010), Stochastic joint inversion of 2d seismic and seismoelectric signals in linear poroelastic materials: A numerical investigation, geophysics, 75(1), N19-N31, doi:10.1190/1.3279833. 
Johnson, D. L., J. Koplik, and R. Dashen (1987), Theory of dynamic permeability in fluid saturated porous media, J. Fluid. Mech., 176, 379-402.

Jougnot, D., and N. Linde (2013), Self-potentials in partially saturated media: The importance of explicit modeling of electrode effects, Vadose Zone Journal, doi:10.2136/vzj2012.0169.

Jougnot, D., N. Linde, A. Revil, and C. Doussan (2012), Derivation of soil-specific streaming potential electrical parameters from hydrodynamic characteristics of partially saturated soils, Vadose Zone J., doi:10.2136/vzj2011.0086.

Jouniaux, L. (2011), Electrokinetic techniques for the determination of hydraulic conductivity, in Hydraulic Conductivity - Issues, Determination and Applications, Lakshmana Elango (Ed.), In Tech Publisher, Rijeka, ISBN 978-953-307-288-3, doi:ISBN 978-953-307-565-5.

Jouniaux, L., and C. Bordes (2012), Frequency-Dependent Streaming Potentials: A Review, Int. J. Geophysics, vol.2012(Hindawi Publishing Corporation), Article ID 648,781, 11 p., doi: $10.1155 / 2012 / 648781$.

Jouniaux, L., and T. Ishido (2012), Electrokinetics in Earth Sciences: a tutorial, Int. J. Geophysics, vol. 2012(Hindawi Publishing Corporation), Article ID 286,107, doi:10.1155/2012/286107.

Jouniaux, L., and J.-P. Pozzi (1995), Permeability dependence of streaming potential in rocks for various fluid conductivity, Geophys. Res. Lett., 22, 485-488.

Jouniaux, L., and J.-P. Pozzi (1997), Laboratory measurements anomalous 0.1-0.5 Hz streaming potential under geochemical changes: Implications for electrotelluric precursors to earthquakes, J. Geophys. Res., 102, 15,335-15,343.

Jouniaux, L., and F. Zyserman (2015), Reply to comment by A. Revil on seismo-electrics, electroseismics, and seismo-magnetics for earth sciences by L. Jouniaux and F. Zyserman, Solid Earth Discussions, 7, C1274.

Jouniaux, L., and F. Zyserman (2016), A review on electrokinetically induced seismo-electrics, electroseismics, and seismo-magnetics for Earth sciences, Solid Earth, 7, 1-36, doi:10.5194/se-7-1-2016.

Jouniaux, L., S. Lallemant, and J. Pozzi (1994), Changes in the permeability, streaming potential and resistivity of a claystone from the Nankai prism under stress, Geophys. Res. Lett., 21, 149-152. 
Jouniaux, L., J.-P. Pozzi, J. Berthier, and P. Massé (1999), Detection of fluid flow variations at the Nankai trough by electric and magnetic measurements in boreholes or at the seafloor, J. Geophys. Res., 104, 29,293-29,309.

Jouniaux, L., M.-L. Bernard, M. Zamora, and J.-P. Pozzi (2000), Streaming potential in volcanic rocks from Mount Peleé, J. Geophys. Res., 105, 8391-8401.

Jouniaux, L., M. Zamora, and T. Reuschlé (2006), Electrical conductivity evolution of non-saturated carbonate rocks during deformation up to failure, Geophys. J. Int., 167, 1017-1026, doi:10.1111/j.1365246X.2006.03136.x.

Jouniaux, L., A. Maineult, V. Naudet, M. Pessel, and P. Sailhac (2009), Review of self-potential methods in hydrogeophysics, C.R. Geoscience, 341, 928-936, doi:10.1016/j.crte.2009.08.008.

Kabir, M., S. Ji, J. Lee, and Y. Koh (2015), Feasibility of streaming potential signal on estimation of solute transport characteristics, J. Soil Groundw. Environ., 20, 41-46, doi:10.7857/JSGE.2015.20.2.041.

Kim, J., M. Nam, and T. Matsuoka (2013), Estimation of $\mathrm{CO}_{2}$ saturation during both $\mathrm{CO}_{2}$ drainage and imbibition processes based on both seismic velocity and electrical resistivity measurements, Geophys. J. Int., electronical access July 9th, doi:10.1093/gji/ggt232.

Li, S., D. Pengra, and P. Wong (1995), Onsager's reciprocal relation and the hydraulic permeability of porous media, Physical Review E, 51(6), 5748-5751.

Linde, N., D. Jougnot, A. Revil, S. K. Matthai, D. Renard, and C. Doussan (2007), Streaming current generation in two-phase flow conditions, Geophys. Res. Lett., 34, LO3306, doi: 10.1029/2006GL028878.

Lorne, B., F. Perrier, and J.-P. Avouac (1999), Streaming potential measurements. 1. properties of the electrical double layer from crushed rock samples, J. Geophys. Res., 104(B8), 17,857-17,877.

Luong, D., and R. Sprik (2014), Examination of a theoretical model of streaming potential coupling coefficient, International Journal of Geophysics, 2014, ID471,819, doi:10.1155/2014/471819.

Maineult, A. (2016), Estimation of the electrical potential distribution along metallic casing from surface self-potential profile, Journal of Applied Geophysics, 129, 66-78. 
Maineult, A., Y. Bernabé, and P. Ackerer (2004), Electrical response of flow, diffusion and advection in a laboratory sand box, Vadose Zone J., (3), 1180-1192.

Maineult, A., Y. Bernabé, and P. Ackerer (2005), Detection of advected concentration and ph fronts from self-potential measurements, J. Geophys. Res., 110, B11,205, doi:10.1029/2005JB003824.

Maineult, A., Y. Bernabé, and P. Ackerer (2006a), Detection of advected, recating redox fronts from self-potential measurements, J. Contaminant Hydrology, (86), 32-52.

Maineult, A., L. Jouniaux, and Y. Bernabé (2006b), Influence of the mineralogical composition on the self-potential response to advection of kcl concentration fronts through sand, Geophys. Res. Lett., 33, L24,311, doi:10.1029/2006GL028048.

Matsushima, M., Y.Honkura, N. Oshiman, S. Baris, M. Tuncer, S. Tank, C. Celik, F. Takahashi, M. Nakanishi, R. Yoshimura, R. Pektas, T. Komut, E. Tolak, A. Ito, Y. Iio, and A. Isikara (2002), Seismoelectromagnetic effect associated with the Izmit earthquake and its aftershocks, Bulletin of the Seismological society of America, 92, 350-360.

Matsushima, N., T. Kikuchi, T. Tosha, S. Nakao, Y. Yano, T. Ishido, K. Hatakeyama, and K. Ariki (2000), Repeat sp measurements at the sumikawa geothermal field, japan, Proc. World Geothermal Congress, Beppu-Morioka, Japan, pp. 2725-2730.

Mauri, G., G. Williams-Jones, and G. Saracco (2010), Depth determinations of shallow hydrothermal system by self-potential and multi-scale wavelet tomography, J. Volc. Geotherm. Res., 191, 233-244.

Mboh, C. M., J. A. Huisman, E. Zimmermann, and H. Vereecken (2012), Coupled hydrogeophysical inversion of streaming potential signals for unsaturated soil hydraulic properties, Vadose Zone J., doi:10.2136/vzj2011.0115.

Mitarai, N., and F. Nori (2006), Wet granular materials, Advances in Physics, 55, 1-50, doi: $10.1080 / 00018730600626065$.

Monachesi, L., F. Zyserman, and L. Jouniaux (2017), A simple model to analytically assess the sh seismoelectric response of the wadose zone, Biot Conference on Poromechanics VI, ASCE publications, 1629. 
Monetti, A., A. Troiano, M. D. Giuseppe, C. Troise, G. D. Natale1, and G. Perillo (2014), Modeling self-potential effects during reservoir stimulation in enhanced geothermal systems, Excerpt from the Proceedings of the 2014 COMSOL Conference in Cambridge, p. 5 p.

Mualem, Y. (1976), A new model for predicting the hydraulic conductivity of unsaturated porous media, Water Resour. Res., 12, 513-522.

Munch, F., and F. Zyserman (2016), Detection of Non-Aqueous Phase Liquids Contamination by SH-TE Seismoelectrics: a Computational Feasibility Study, Journal of Applied Geophysics, doi: 10.1016/j.jappgeo.2016.03.026.

Onsager, L. (1931), Reciprocal relations in irreversible processes:i, Phys. Rev., 37, 405-426.

Packard, R. G. (1953), Streaming potentials across capillaries for sinusoidal pressure, J. Chem. Phys, 1(21), 303-307.

Parkhomenko, E., and I. Gaskarov (1971), Borehole and laboratory studies of the seismoelectric effect of the second kind in rocks, Izv. Akad. Sci. USSR, Physics Solid Earth, 9, 663-666.

Parkhomenko, I., and C. Tsze-San (1964), A study of the influence of moisture on the magnitude of the seismoelectric effect in sedimentary rocks by a laboratory method, Bull. (Izv.) Acad. Sci., USSR, Geophys. Ser., pp. 115-118.

Parks, G. (1965), The isoelectric points of solid oxides, solid hydroxides, and aqueous hydroxo-complex, Chem. Rev., 65, 177-198.

Pengra, D. B., S. X. Li, and P.-Z. Wong (1999), Determination of rock properties by low frequency ac electrokinetics, J. Geophys. Res., 104(B12), 29.485-29.508.

Perrier, F., and T. Froidefond (2003), Electrical conductivity and streaming potential coefficient in a moderately alkaline lava series, Earth and Planetary Science Letters, 210, 351-363, doi: $10.1016 / \mathrm{S} 0012-821 \mathrm{X}(03) 00105-5$.

Perrier, F., and P. Morat (2000), Characterization of electrical daily variations induced by capillary flow in the non-saturated zone, Pure Appl. Geophys., 157, 785-810.

Pinettes, P., P. Bernard, F. Cornet, G. Hovhannissian, L. Jouniaux, J.-P. Pozzi, and V. Barthés (2002), On the difficulty of detecting streaming potentials generated at depth, Pure Appl. Geophys., 159, 
Pozzi, J.-P., and L. Jouniaux (1994), Electrical effects of fluid circulation in sediments and seismic prediction, C.R. Acad. Sci. Paris, serie II, 318(1), 73-77.

Pride, S. (1994), Governing equations for the coupled electromagnetics and acoustics of porous media, Phys. Rev. B: Condens. Matter, 50, 15,678-15,695.

Pride, S., and F. D. Morgan (1991), Electrokinetic dissipation induced by seismic waves, Geophysics, 56(7), 914-925.

Rangarajan, R., D. Muralidharan, and S. Chandra (2014), Time lapse tracer and sp measurements to characterize the hydrodynamics of fractured granite aquifer: A case study, JOURNAL GEOLOGICAL SOCIETY OF INDIA, 83, 681-687.

Reeves, P., and M. Celia (1996), A functional relationship between capillary pressure, saturation, and interfacial area as revealed by a pore-scale network model, Water Resources Research, 32, 2345-2358.

Ren, H., Q. Huang, and X. Chen (2010), A new numerical technique for simulating the coupled seismic and electromagnetic waves in layered porous media, Earthquake Science, 23(2), 167-176.

Reppert, P. M., F. D. Morgan, D. P. Lesmes, and L. Jouniaux (2001), Frequency-dependent streaming potentials, J. Colloid Interface Sci., 234, 194-203, doi:10.1006/jcis.2000.7294.

Revil, A., and A. Cerepi (2004), Streaming potentials in two-phase flow conditions, Geophys. Res. Lett., 31, L11,605, doi:10.1029/2004GL020140.

Revil, A., and P. Glover (1998), Nature of surface electrical conductivity in natural sands, sandstones, and clays, Geophys. Res. Lett., 25(5), 691-694.

Revil, A., and A. Jardani (2010), Seismoelectric response of heavy oil reservoirs: theory and numerical modelling, Geophys. J. Int., 180, 781-797, doi:10.1111/j.1365-246X.2009.04439.x.

Revil, A., and N. Linde (2011), Comment on "streaming potential dependence on water-content in fontainebleau sand" by A. Revil and N. Linde, Geophys. J. Int.

Revil, A., and H. Mahardika (2013), Coupled hydromechanical and electromagnetic disturbances in unsaturated porous materials, Water Resour. Res., 49, 744-766. 
Revil, A., N. Linde, A. Cerepi, D. Jougnot, S. Matthäi, and S. Finsterle (2007), Electrokinetic coupling in unsaturated porous media, J. Colloid Interface Sci., 313, 315-327.

Richards, L. (1931), Capillary conduction of liquids through porous mediums, Physics, 1, 318-333.

Rutgers, A. (1940), Streaming effects and surface conduction. streaming potentials and surface conductance, Trans. Faraday Soc., 35, 69-80, doi:10.1039/TF9403500069.

Sailhac, P., M. Darnet, and G. Marquis (2004), Electrical streaming potential measured at the ground surface: forward modeling and inversion issues for monitoring infiltration and characterizing the vadose zone, Vadose Zone J., (3), 1200-1206.

Saunders, J. H., M. D. Jackson, and C. C. Pain (2008), Fluid flow monitoring in oilfields using downhole measurements of electrokinetic potential, Geophysics, 73, E165-E180, doi:10.1190/1.2959139.

Sava, P., A. Revil, and M. Karaoulis (2014), High definition cross-well electrical resistivity imaging using seismoelectric focusing and image-ghided inversion, Geophys. J. Int., 198, 880-894, doi: 10.1093/gji/ggu166.

Schakel, M., D. Smeulders, E. Slob, and H. Heller (2012), Seismoelectric fluid/porous-medium interface response model and measurements, Transport in Porous media, 93, 271-282, doi:10.1007/s11242-0119869-8.

Schoemaker, F., D. Smeulders, and E. Slob (2007), Simultaneous determination of dynamic permeability and streaming potential, SEG expanded abstracts, 26, 1555-1559.

Schoemaker, F., D. Smeulders, and E. Slob (2008), Electrokinetic effect: Theory and measurement, SEG Technical Program Expanded Abstracts, pp. 1645-1649.

Sears, A., and J. Groves (1978), The use of oscillating laminar flow streaming potential measurements to determine the zeta potential of a capillary surface, J. Colloid Interface Sci., 65, 479-482.

Sénéchal, P., S. Garambois, and C. Bordes (2010), Feasibility of acoustic imaging for in-situ characterization of subsurface soil injected with fresh mortar, J. appl. Geophys., 72(3), 184193.

Sposito, G. (1989), The chemistry of soils, Oxford University, Oxford.

Strahser, M., L. Jouniaux, P. Sailhac, P.-D. Matthey, and M. Zillmer (2011), Dependence of seismoelectric amplitudes on water-content, Geophys. J. Int., 187, 1378-1392. 
Takahashi, M. (2005), Zeta potential of microbubbles in aqueous solutions: electrical properties of the gas-water interface, J. Phys. Chem. B, 109, 21,858-21,864, doi:10.1021/jp0445270.

Tardif, E., P. Glover, and J. Ruel (2011), Frequency-dependent streaming potential of ottawa sand, $J$. Geophys. Res., 116, B04,206, doi:10.1029/2010JB008053.

Teja, A. S., and P. Rice (1981), Generalized corresponding states method for the viscosities of liquid mixtures, Ind. Eng. Chem. Fund., 20(1), 77-81.

Thompson, A., S. Hornbostel, J. Burns, T. Murray, R. Raschke, J. Wride, P. McCammon, J. Sumner, G. Haake, M. Bixby, W. Ross, B. White, M. Zhou, and P. Peczak (2005), Field tests of electroseismic hydrocarbon detection, SEG Technical Program Expanded Abstracts, pp. 565-568.

Thompson, A., J. Sumner, and S. Hornbostel (2007), Electromagnetic-to-seismic conversion: A new direct hydrocarbon indicator, The Leading Edge, 26, 428-435, doi:10.1190/1.2723205.

Titov, K., Y. Ilyin, P. Konosavski, and A. Levitski (2002), Electrokinetic spontaneous polarization in porous media: petrophysics and numerical modelling, J. Hydrol., 267, 207-216, doi:10.1016/S00221694(02)00151-8.

Tsakiroglou, C., and M. Fleury (1999), Pore network analysis of resistivity index for water-wet porous media, Transport in Porous media, 35, 89-128.

Valuri, J., T. Dean, and J. Dupuis (2012), Seismoelectric acquisition in an arid environment, 22nd International Geophysical Conference and Exhibition, 26-29 February.

van Genuchten, M. (1980), Aclosed form equation for predicting the hydraulic conductivity of unsaturated soils, Soil. Sci. Soc. Am. J., 40, 892-898.

van Genuchten, M., and D. Nielsen (1985), On describing and predicting the hydraulic properties of unsaturated soils, Earth and Planetary Science Letters, 3, 615-628.

VanCappellen, P., L. Charlet, W. Stumm, and P.Wersin (1993), A surface complexation model of the carbonate mineral-aqueous solution interface, Geochimica and Cosmochimica Acta, 57, 3505-3518.

Vinogradov, J., and M. Jackson (2011), Multiphase streaming potential in sandstones saturated with gas/brine and oil/brine during drainage and imbibition, Geophys. Res. Lett., 38, L01,301, doi: 10.1029/2010GL045726. 
Vinogradov, J., M. Jaafar, and M. D. Jackson (2010), Measurement of streaming potential coupling coefficient in sandstones saturated with natural and artificial brines at high salinity, J. Geophys. Res., 115, B12,204, doi:10.1029/2010JB007593,2010.

Walker, E., P. Glover, and J. Ruel (2014), A transient method for measuring the dc streaming potential coefficient of porous and fractured rocks, J. Geophys. Res., 119, doi:10.1002/2013JB010579.

Wang, J., and H. Hu (2012), The determination of electrokinetic coupling coefficient and zeta potential of rock samples by electrokinetic measurements, Advanced Materials Research, 516-517, 1870-1873.

Wang, J., H. Hu, and W. Guan (2015a), Experimental measurements of seismoelectric signals in borehole models, Geophys. J. Int., 203, 1937-1945.

Wang, J., H. Hu, W. Guan, and H. Li (2015b), Electrokinetic experimental study on saturated rock samples: zeta potential and surface conductance, Geophys. J. Int., 201, 869-877, doi:10.1093/gji/ggv013.

Wang, J., H. Hu, and W. Guan (2016), The evaluation of rock permeability with streaming current measurements, Geophys. J. Int., 206(3), 1563-1573, doi:10.1093/gji/ggw231.

Warden, S., S. Garambois, L. Jouniaux, D. Brito, P. Sailhac, and C. Bordes (2013), Seismoelectric wave propagation numerical modeling in partially saturated materials, Geophys. J. Int., 194, 1498-1513, doi:10.1093/gji/ggt198.

Yang, C., T. Dabros, D. Li, J. Czarnecki, and J. Masliyah (2001), Measurement of the zeta potential of gas bubbles in aqueous solutions by microelectrophoresis method, J. Colloid Interface Sci., 243, $128-135$.

Zhang, J., J. Vinogradov, and M. D. Jackson (2014), Streaming potential dependence on water saturation during drainage and imbibition, AGU abstracts.

Zhou, Q., J. Shimada, and A. Sato (2002), Three-dimensional spatial and temporal monitoring of soil water content using electrical resistivity tomography, Water Resources Research, 37(2), 273-285.

Zyserman, F., P. Gauzellino, and J. Santos (2012), Numerical evidence of gas hydrate detection by means of electroseismics, J. Applied Geophysics, 86, 98-108.

Zyserman, F., L. Jouniaux, S. Warden, and S. Garambois (2015), Borehole seismoelectric logging using a shear-wave source:Possible application to $\mathrm{CO}_{2}$ disposal?, International Journal of Greenhouse Gas 
Zyserman, F., L. Monachesi, and L. Jouniaux (2017a), Dependence of shear wave seismoelectrics on soil texture: a numerical study in the vadose zone, Geophys. J. Int., 208, 918-935.

Zyserman, F., L. Monachesi, and L. Jouniaux (2017b), Reply to comment by A. Revil on Dependence of shear wave seismoelectrics on soil texture: a numerical study in the vadose zone by F. Zyserman, LB Monachesi and L. Jouniaux, Geophys. J. Int., 210, 1652-1658. 


\begin{tabular}{|l|l|}
\hline Reference & $C_{s 0}\left(S_{w}\right) / C_{\text {sat }}$ \\
\hline Perrier and Morat [2000] & $S_{e}^{2} / S_{w}^{n}$ \\
\hline Guichet et al. [2003] & $S_{e}$ \\
\hline Jackson [2010] & $S_{e}^{(L+2+2 / \lambda)} Q_{r}\left(S_{w}\right) / S_{w}{ }^{n}$ \\
\hline Allègre et al. [2012] & $S_{e}\left(1+32\left(1-S_{e}\right)^{0.4}\right)$ \\
\hline
\end{tabular}

Table 1. Streaming Potential Coefficient behaviours as a function of water-saturation. The effective saturation $S_{e}$ is defined in eq.16 in which $S_{w r}$ denotes the residual saturation, $n$ is Archie's saturation exponent, $\mathrm{L}$ and $\lambda$ are the Mualem's parameters in the relative permeability formula Mualem [1976]. 


\begin{tabular}{|c|c|c|}
\hline Parameter & $S_{w}$ dependence & Expression \\
\hline$K_{f}(\mathrm{~Pa})$ & YES & $\begin{array}{l}\text { Brie et al. [1995]: } \\
K_{f}\left(S_{w}\right)=\left(K_{\text {eau }}-K_{\text {air }}\right) S_{w}^{5}+K_{\text {air }}\end{array}$ \\
\hline$\rho_{f}\left(\mathrm{Kg} / \mathrm{m}^{3}\right)$ & YES & $\begin{array}{l}\text { Arithmetic average: } \\
\rho_{f}\left(S_{w}\right)=\left(1-S_{w}\right) \rho_{a i r}+S_{w} \rho_{e a u}\end{array}$ \\
\hline$\eta(\mathrm{Pa} . \mathrm{s})$ & YES & $\begin{array}{l}\text { Teja and Rice }[1981] \text { : } \\
\eta\left(S_{w}\right)=\eta_{a i r}\left(\eta_{\text {eau }} / \eta_{a i r}\right)^{S_{w}}\end{array}$ \\
\hline$b_{1}(\mathrm{~N} . \mathrm{s} / \mathrm{m})$ & NO & Computed for water only with Einstein-Stokes' law. \\
\hline$\epsilon(\mathrm{F} / \mathrm{m})$ & YES & $\begin{array}{l}\text { CRIM: } \\
\epsilon=\epsilon_{0}\left[(1-\phi) \sqrt{\kappa_{\text {quartz }}}+\phi S_{w} \sqrt{\kappa_{\text {eau }}}+\phi\left(1-S_{w}\right) \sqrt{\kappa_{\text {air }}}\right]^{2}\end{array}$ \\
\hline$\alpha_{\infty}$ & NO & Hydraulic (geometric) tortuosity. \\
\hline$\Lambda(\mathrm{m})$ & NO & Characteristic length of the microstucture. \\
\hline$\omega_{t}(\mathrm{rad} / \mathrm{s})$ & YES & $\omega_{t}=\frac{\eta\left(S_{w}\right)}{F k_{0} \rho_{f}\left(S_{w}\right)}$ \\
\hline$C_{e m}$ and $C_{o s}(S)$ & NO & Computed for water only using Pride's expressions. \\
\hline$\sigma(\mathrm{S} . \mathrm{m})$ & YES & $\sigma\left(S_{w}, \omega\right)=\frac{1}{F}\left(\sigma_{f} S_{w}^{n}+2 \frac{C_{e m}+C_{o s}(\omega)}{\Lambda}\right)$ \\
\hline
\end{tabular}

Table 2. Effective properties for a water-air mixture, from Warden et al. [2013] 
20 respectively.

\begin{tabular}{ccccccccccc}
\hline \multicolumn{1}{c}{ Experiment } & $n$ & $m_{\theta}$ & $m_{K}$ & $\lambda$ & $\alpha_{v}\left[\mathrm{~cm}^{-1}\right]$ & $K_{s}[\mathrm{~cm} / \mathrm{s}]$ & $L$ & $h_{a}[\mathrm{~cm}]$ & $\theta_{s}$ & $\theta_{r}$ \\
\hline$[$ Linde et al., 2007] & 10 & 0.9 & 0.87 & $/$ & 0.0151 & 0.0068 & 0.5 & $/$ & 0.34 & 0.05 \\
\hline$[$ Allègre et al., 2010] & $/$ & $/$ & $/$ & 3.88 & $/$ & 0.0017 & 0.5 & 40 & 0.355 & 0.11 \\
\hline$[$ Mboh et al., 2012] & 3.879 & $/$ & $/$ & $/$ & 0.02 & 0.0031 & 0.5 & $/$ & 0.33 & $0.01-0.1$ \\
\hline [Allègre et al., 2014] & 4.35 & $/$ & $/$ & $/$ & 0.022 (drainage) & 0.0041 & 0.5 & $/$ & 0.41 & 0.01
\end{tabular}
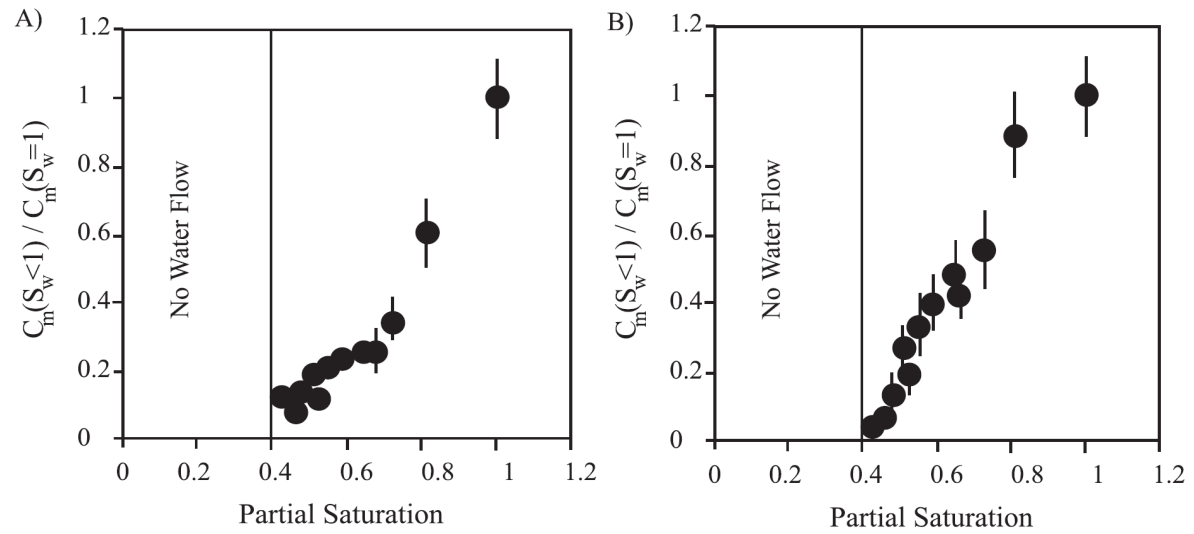

Table 3. Parameters used in eqs. 19, 20, 21 and 22. The parameters $m_{\text {theta }}$ and $m_{K}$ are the values of $m$ used in eqs. 19 and

Figure 1. a) Normalized streaming potential coefficient $C_{s 0}\left(S_{w}\right) / C_{s 0}\left(S_{w}=1\right)$ versus water saturation. The zeta-potential is assumed to be insensitive to water saturation, and is calculated using equation 17 . The gas injected is argon. The experimental results are corrected for $\mathrm{pH}$ and fluid electrical conductivity. Variations in zeta-potential are corrected to a $\mathrm{pH}$ value of 8 and a fluid electrical conductivity value of of $140 \mathrm{mS} / \mathrm{cm}$, using $\zeta=-0.0146 \ln \left(\sigma_{f}\right)-0.0854$ (from Guichet et al. [2003]). b) Normalized streaming potential coefficient $C_{s 0}\left(S_{w}\right) / C_{s 0}\left(S_{w}=1\right)$ versus water saturation as in a), using $\zeta=20 \log _{10}\left(\sigma_{f}\right)$ (from Guichet et al. [2003]) 

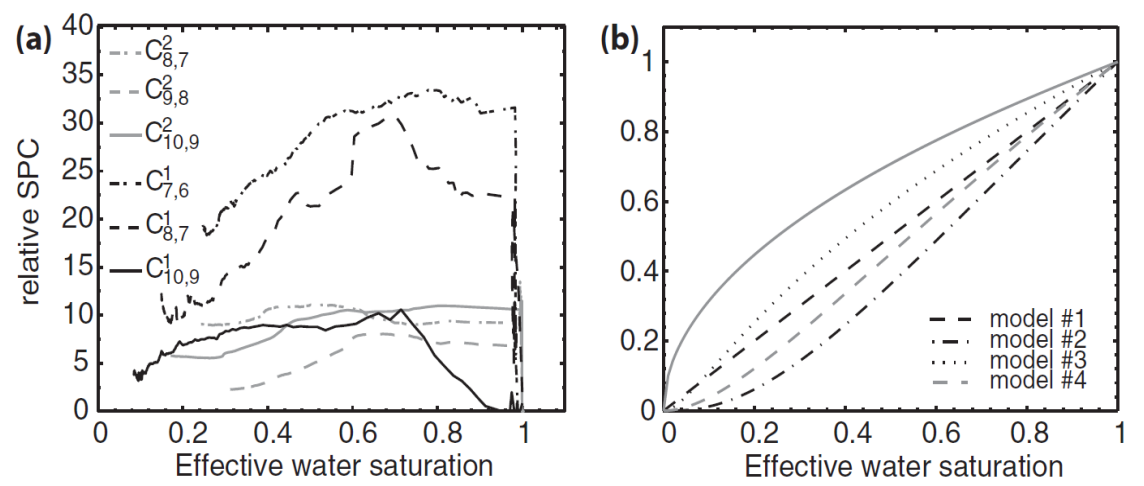

Figure 2. a) SPC measured during a drainage of a sand, on 6 dipoles with electrodes distance of $10 \mathrm{~cm}$, showing a nonmonotoneous behaviour as a function of the water saturation (from Allègre et al. [2010]); b) current models from Guichet et al. [2003] (black dashed), Perrier and Morat [2000] (black dotted), and Revil et al. [2007] (grey dotted dashed). Note the difference in Y-axis scale.

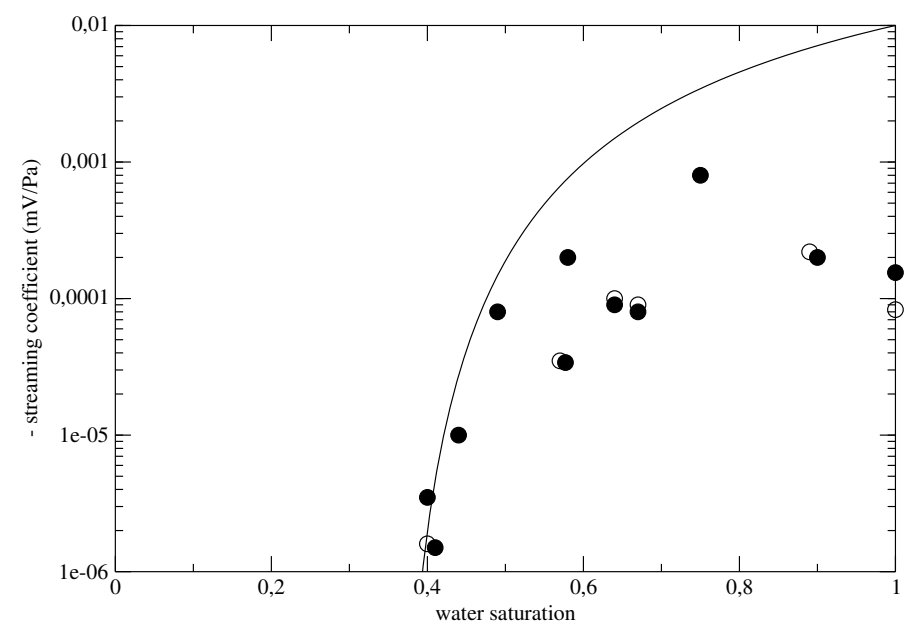

Figure 3. Streaming potential coefficient measured for sample E3 (black circles) and E39 (empty circles) from Revil et al. [2007] (Fig. 7b) including measurements at full saturation (=1) not reported by Revil et al. [2007] and shown in Revil and Cerepi [2004] (Fig. 3) on same samples with the same water conductivity $0.93 \mathrm{~S} / \mathrm{m}$. The model from Revil et al. [2007] (continuous line) with $n=2.7 ; S_{w}^{r}=0.36 ; \lambda=0.87$, eq.112 and 113 calculated with the value at saturation extrapolated from the values obtained at various saturation to $10^{-2} \mathrm{mV} / \mathrm{Pa}$, which is two orders of magnitude above the measured values. From Allègre et al. [2011] 


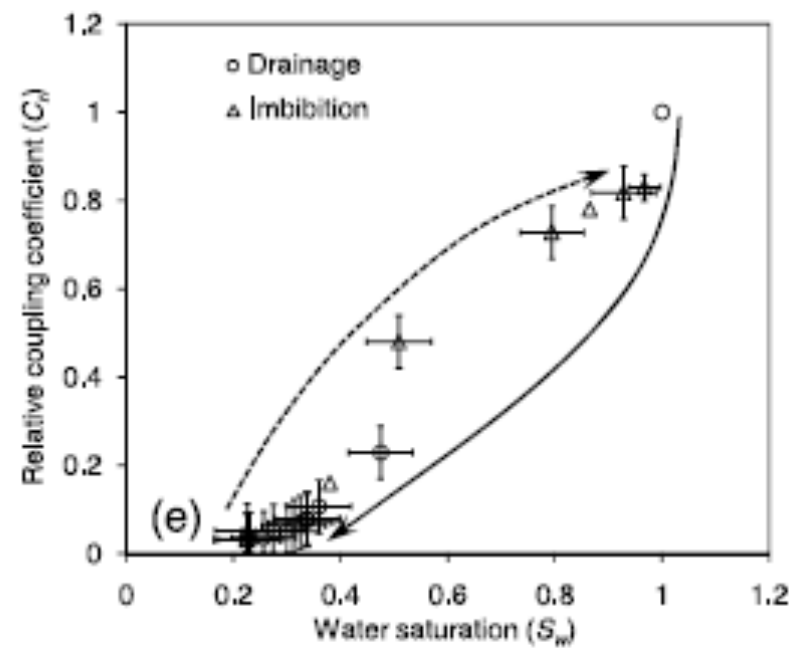

Figure 4. Streaming potential measured with brine/nitrogen on St Bees sample by Vinogradov and Jackson [2011] (fig. 3e).

For the drainage no measured values are available between 0.5 water-saturation and full saturation.

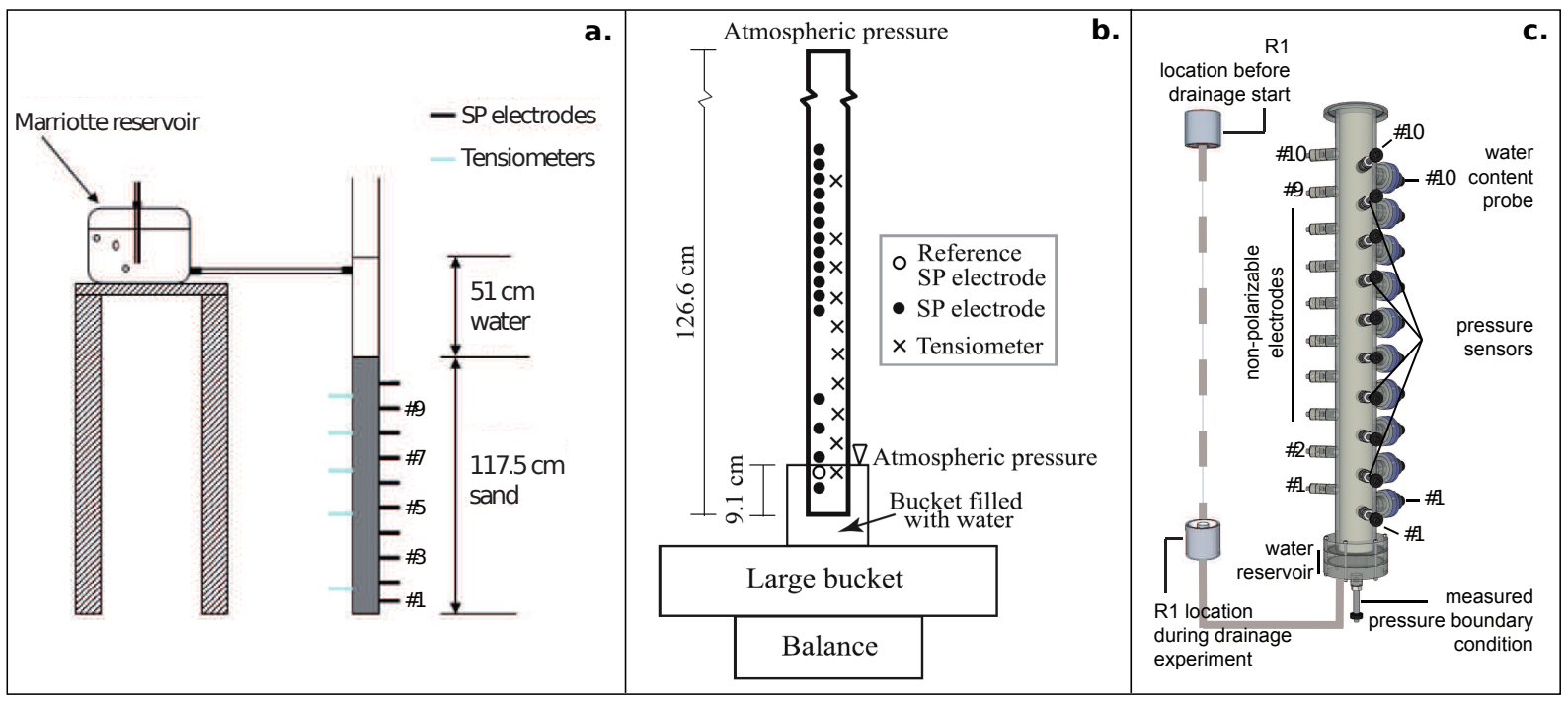

Figure 5. Experimental apparatus from Mboh et al. [2012] (a), from Linde et al. [2007] (b) and from Allègre et al. [2010]

(c). 

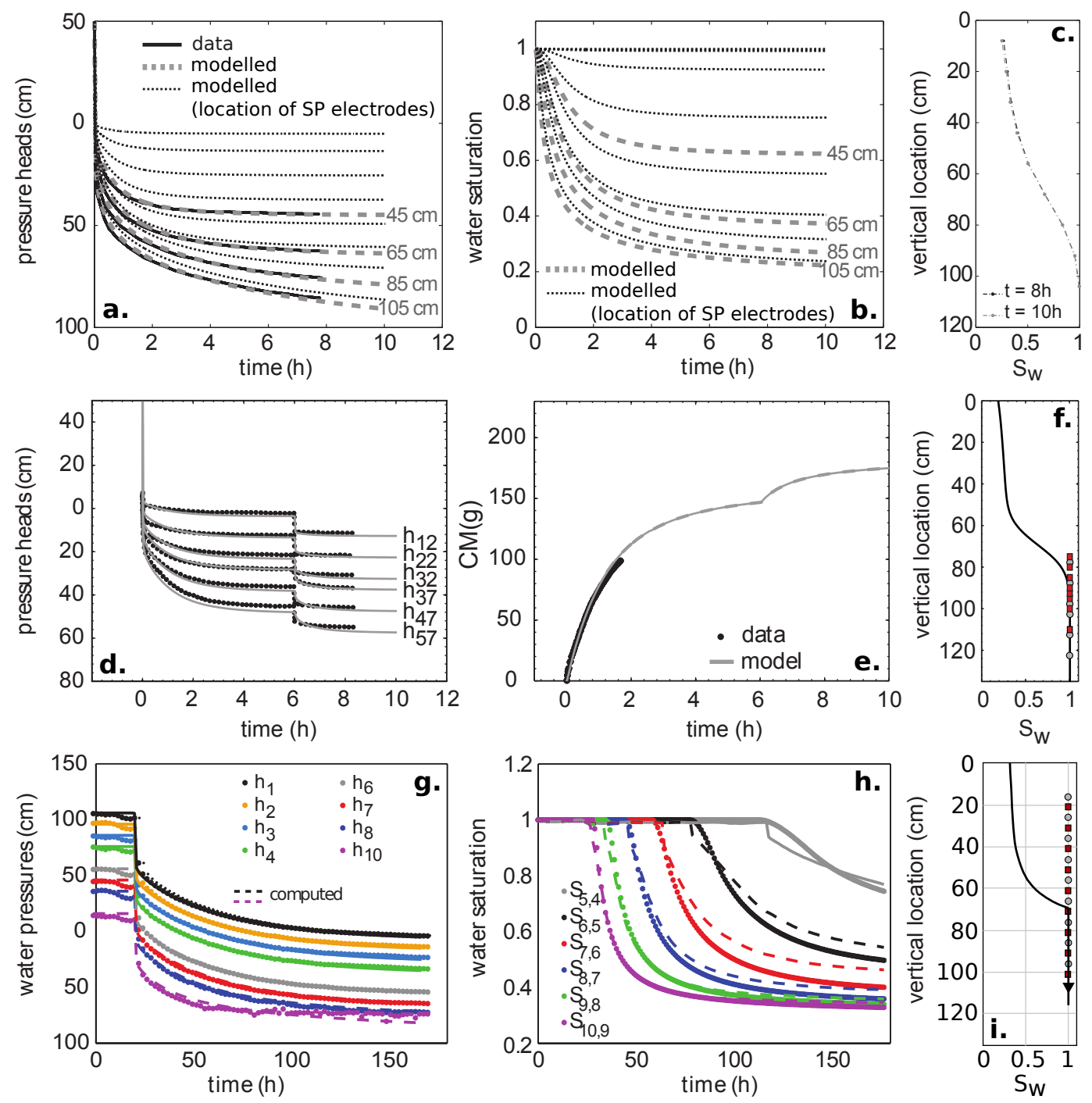

Figure 6. Measured and computed pressure heads time-series, water saturation time-series and vertical saturation profiles for (a-c) Mboh et al. [2012] and (g-i) Allègre et al. [2010] experiments. The measured and computed cumulative outflow are shown instead of saturations for Linde et al. [2007] study (d-f). Pressure heads are both computed at the locations of tensiometers and SP electrodes for Mboh et al. [2012] experiment (b). The vertical saturation profiles are inferred from computed saturations at the end of each experiment. Red squares and gray circles indicate the locations of SP electrodes and tensiometers respectively. 

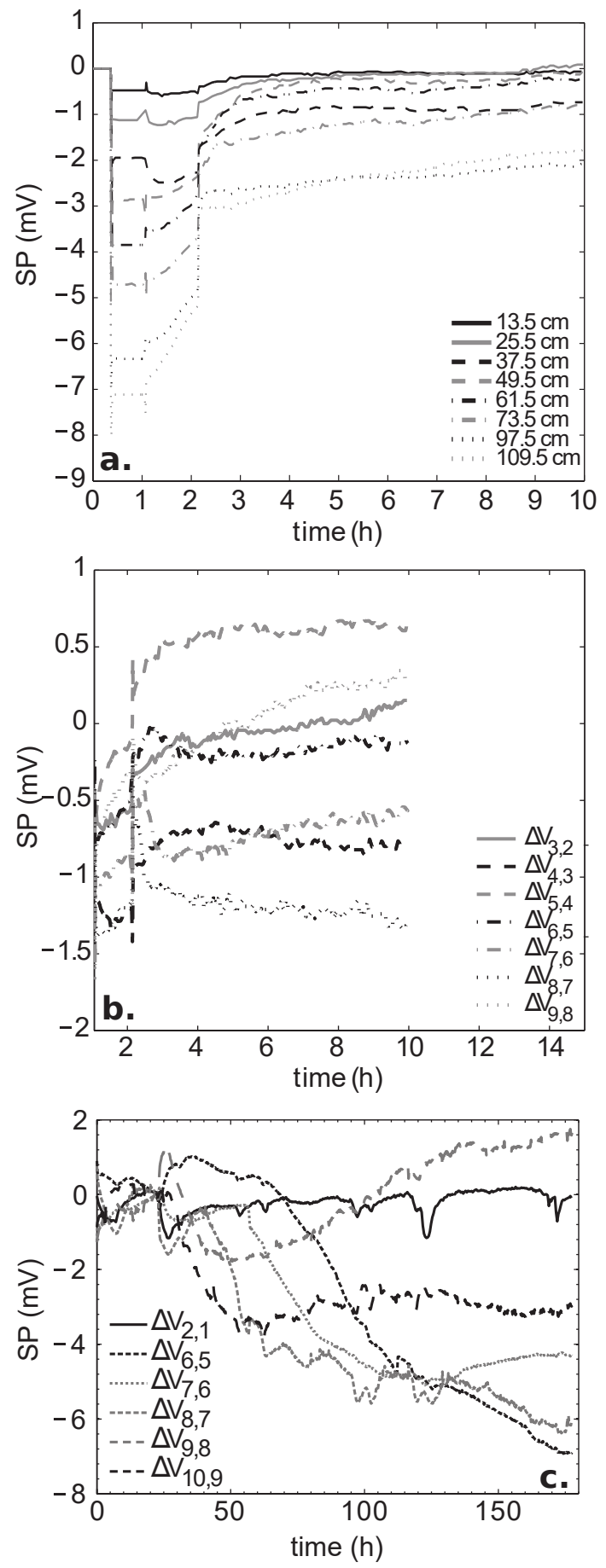

Figure 7. (a) Raw SP measurements during the drainage experiment from Mboh et al. [2012] shifted to zero at $t=0$. (b) SP differences computed for couples of consecutive electrodes. (c) SP differences computed for couples of consecutive to 10 from the bottom to the top of the column. 

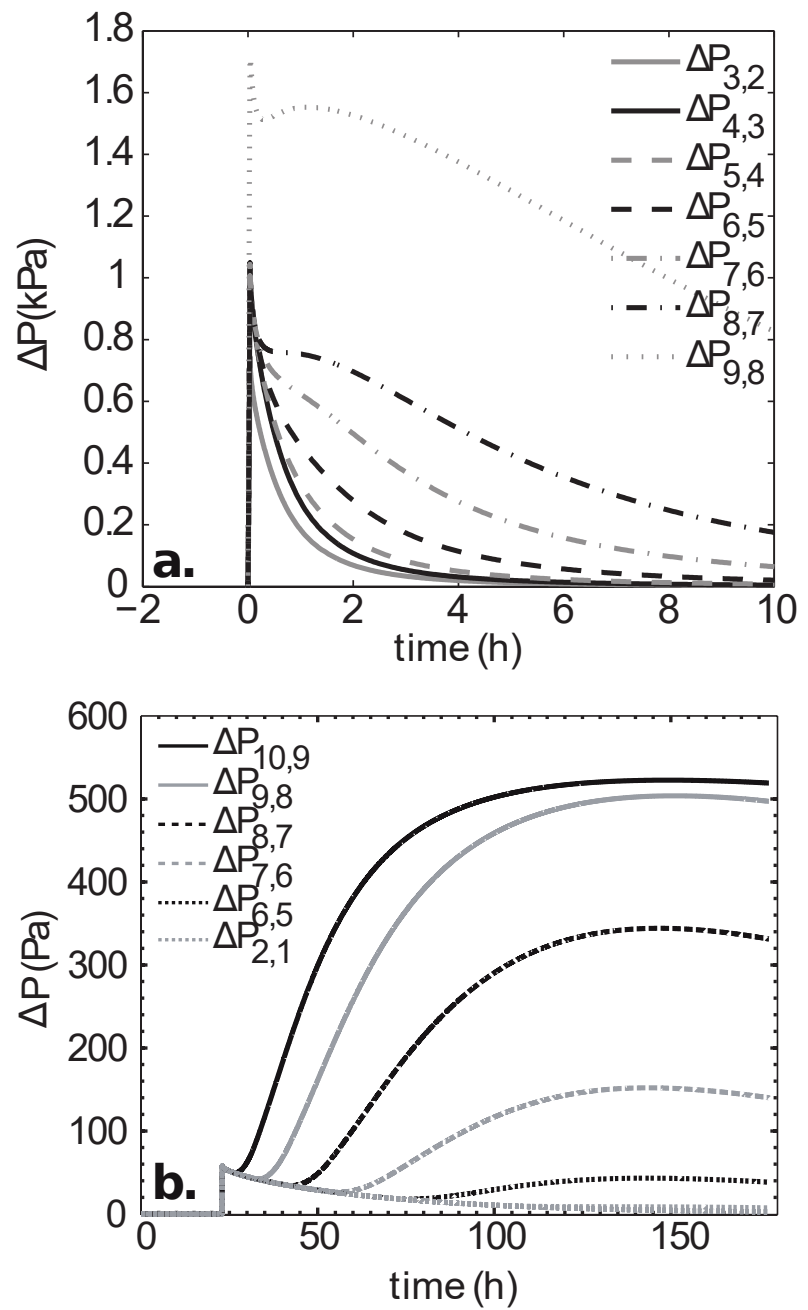

Figure 8. Total water pressure differences $\Delta P$ inferred from computed $h$ time-series (fig. 6) for (a) Mboh et al. [2012] and (b) Allègre et al. [2010] drainage experiments. Each each curve corresponds to the pressure difference at the locations of two consecutive SP electrodes (fig. 5). 

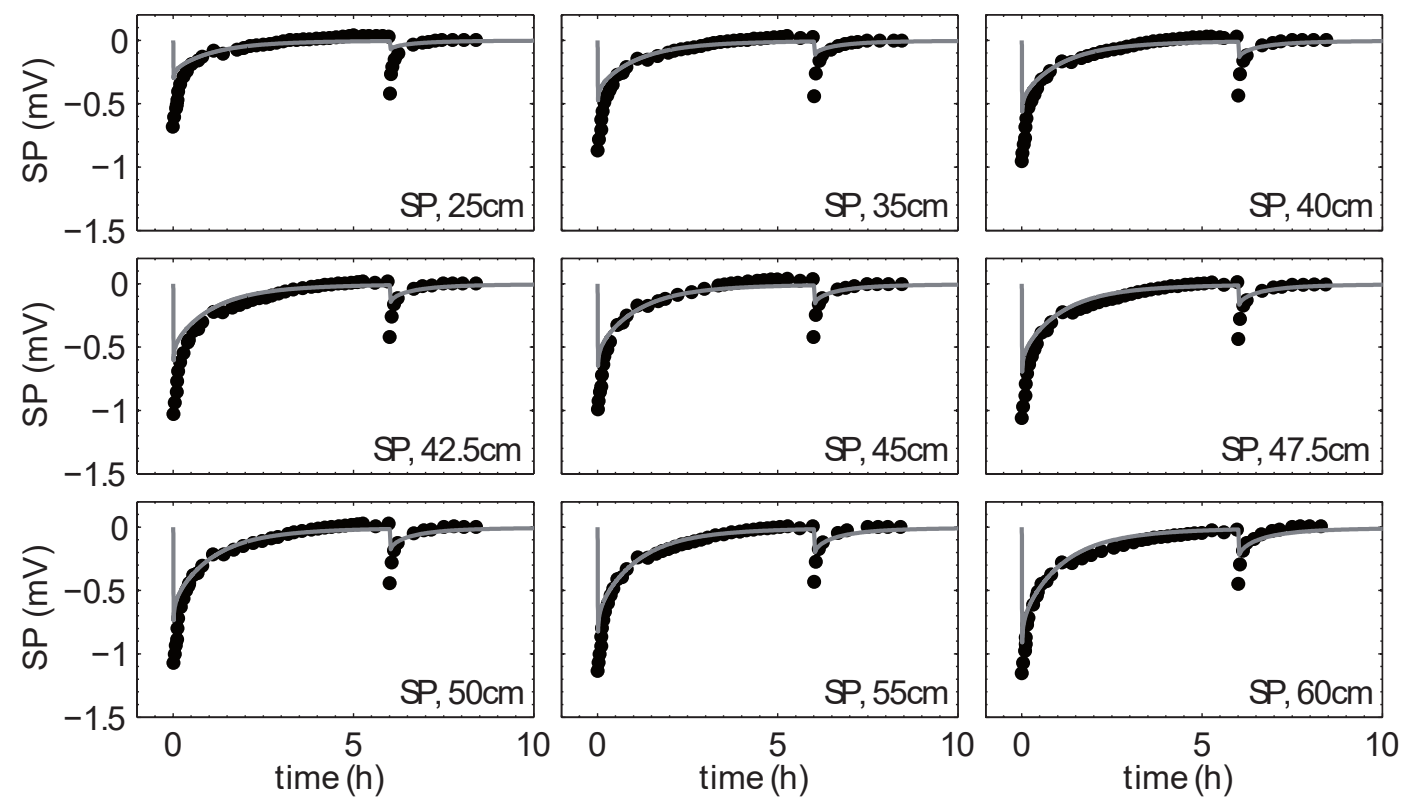

Figure 9. Measured SP from Linde et al. [2007] during the drainage experiment (black dots) : the first stage of the drainage experiment consisted of draining the saturated sand column where the lower boundary condition consisted of a fixed head, the second stage of the experiment began after six hours when the lower boundary was removed to ensure a free drainage. Simulated SP (gray line) calculated using the procedure from Allègre et al. [2012] with a constant SPC $\left(C_{\text {sat }}\right)$. Despite a constant SPC, the measurements are fairly reproduced regardless the dipole location within the column. This good fit is explained by the limited saturation range $\left(0.85<S_{w}<1\right)$ investigated during the experiment (see text for details). 


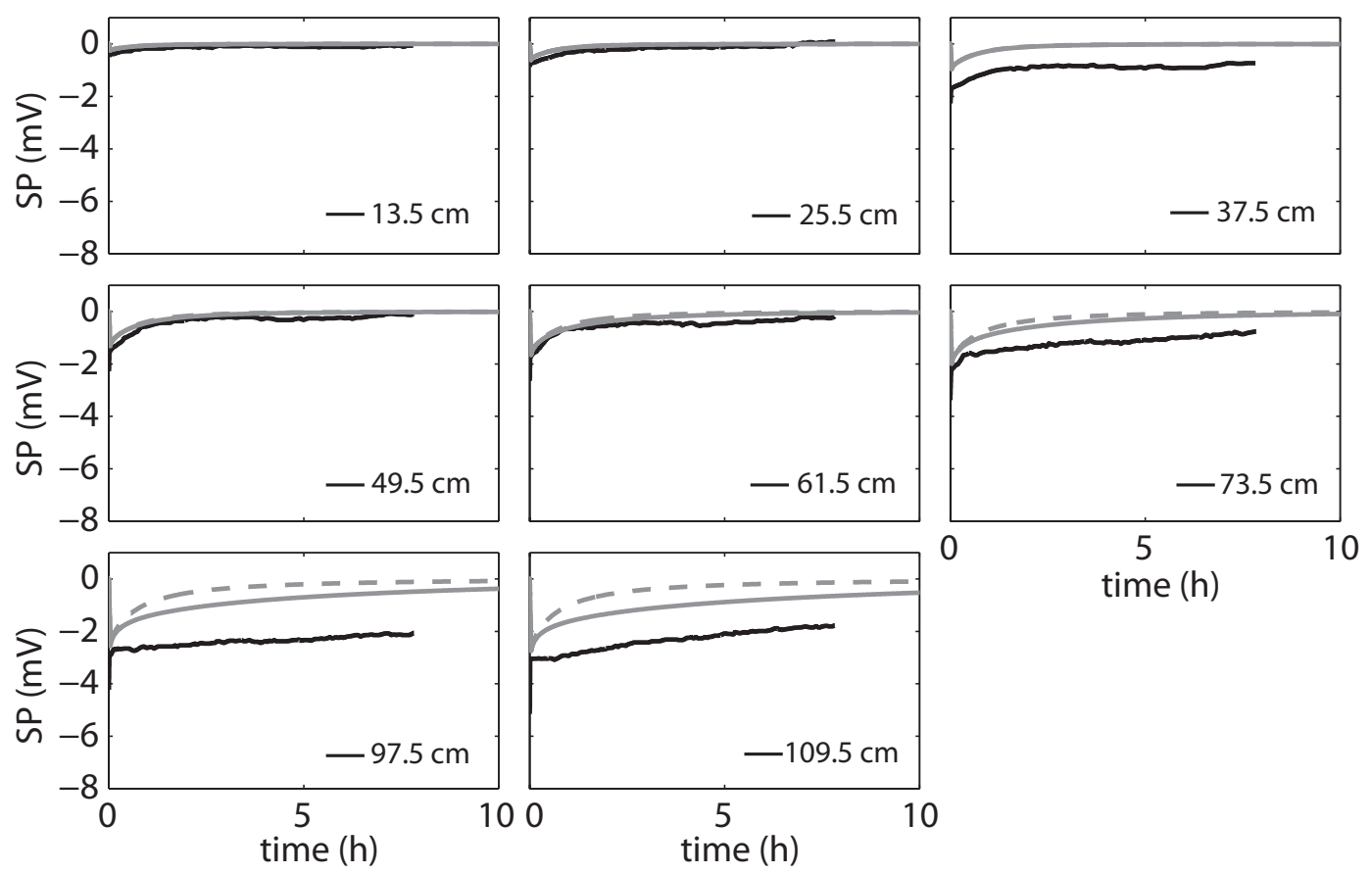

Figure 10. Measured SP differences from Mboh et al. [2012] drainage experiment (black) inferred from raw signals (see text for details), and simulated (gray) using the procedure from Allègre et al. [2012] with a constant SPC ( $C_{\text {sat }}$, dashed line) and the SPC model from Guichet et al. [2003] (solid line). The measurements are well reproduced in terms of both shape and amplitude for lower location within the column where the range of saturation is limited. A simple SPC model does seem well suited for all the dipoles. 


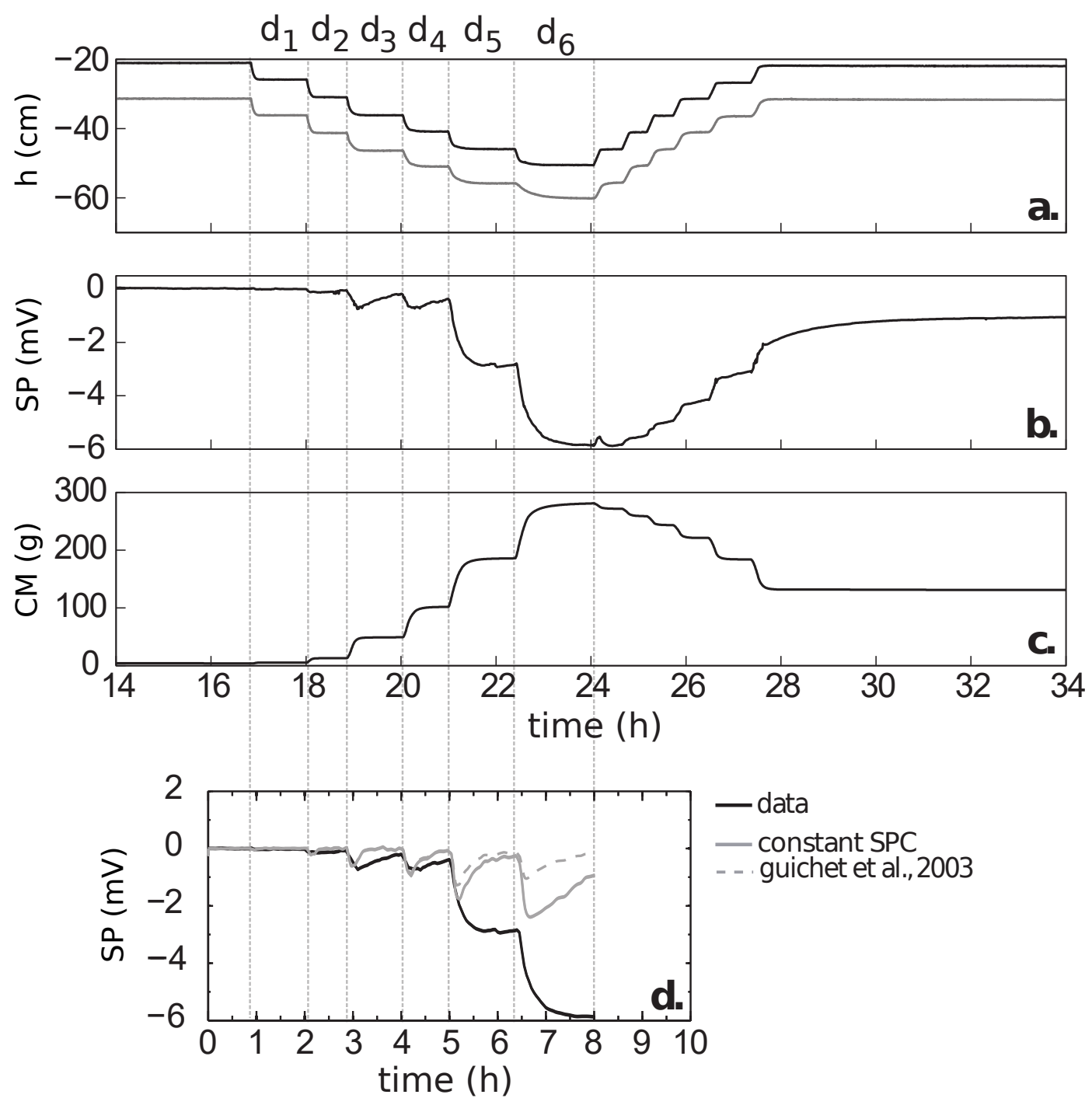

Figure 11. Step-by-step drainage and imbibition experiment performed with the experimental setup from Allègre et al. [2014]. The drainage phases are triggered by decreasing the pressure boundary conditions by $5 \mathrm{~cm}$ pressure steps. (a) Measured pressure heads, (b) measured self-potentials, (c) measured cumulative outflow. (d) Close-up view on the six drainage phases, noted $d_{1}$ to $d_{6}$. 

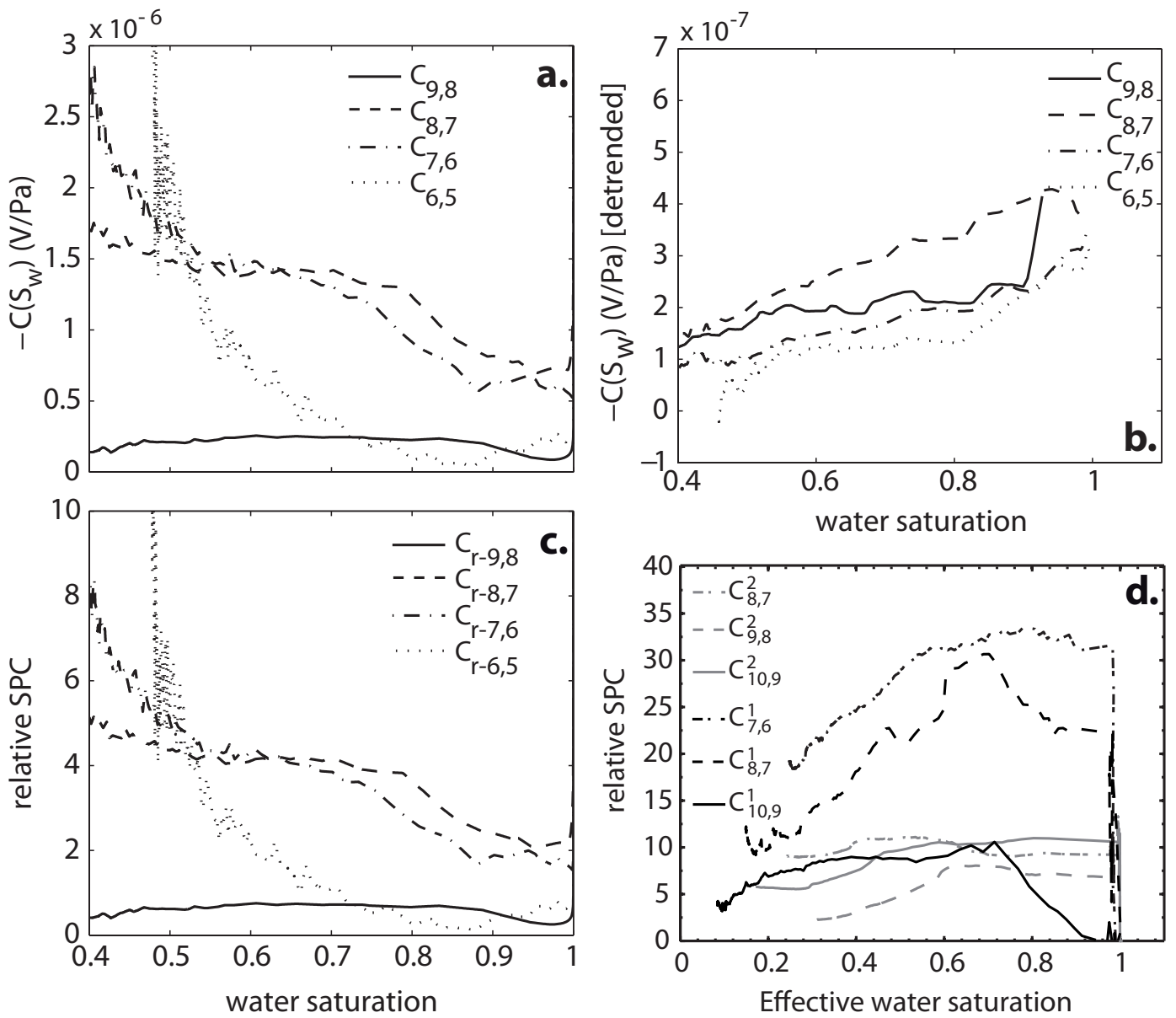

Figure 12. Streaming potential coefficient curves inferred from Mboh et al. [2012] dataset using (a) uncorrected SP differ- 


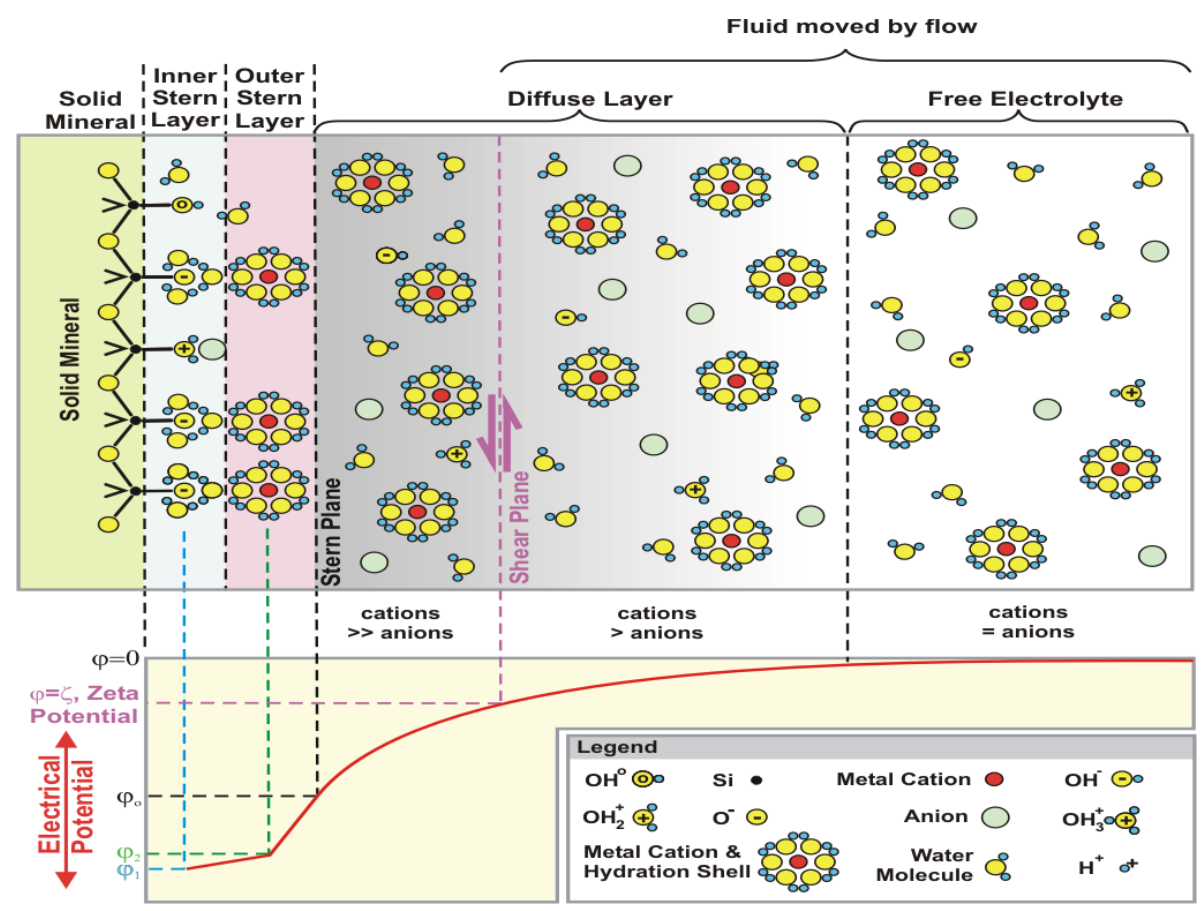

Figure 13. Electric double layer, first published in [Glover and Jackson, 2010]. The solid mineral presented is the case of silica. At $p H$ above the isoelectric point the cations are adsorbed within the Stern layer; there is an excess of cations in the diffuse layer. The zeta potential is defined at the shear plane. The fluid flow creates a streaming current which is balanced by the conduction current, leading to the streaming potential. 


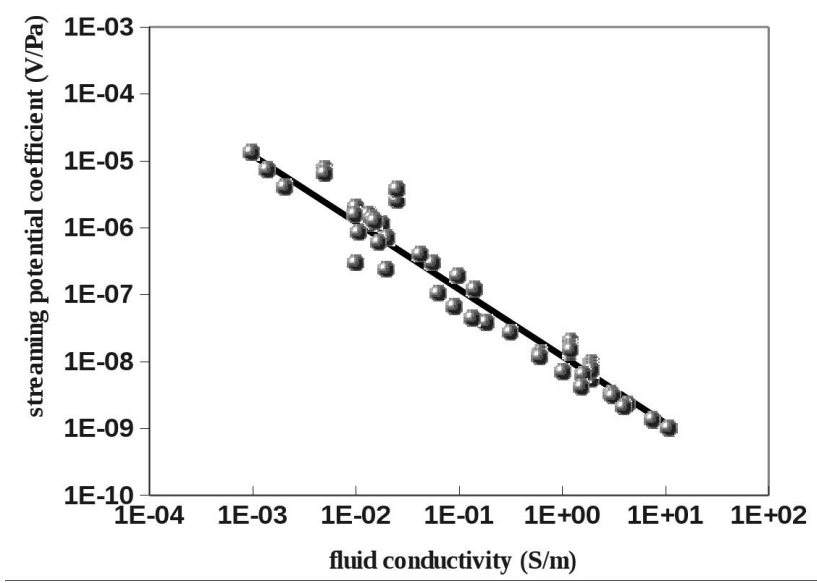

Figure 14. Streaming potential coefficient from data collected (in absolute value) on sands and sandstones at $\mathrm{pH}$ 7-8 (when available) from Ahmad [1964]; Li et al. [1995]; Jouniaux and Pozzi [1997]; Lorne et al. [1999]; Pengra et al. [1999]; Guichet et al. [2003]; Perrier and Froidefond [2003]; Guichet et al. [2006]; Ishido and Mizutani [1981]; Jaafar et al. [2009]. The regression (black line) leads to $C_{s 0}=-1.2 \times 10^{-8} \sigma_{f}^{-1}$. A zeta potential of $-17 \mathrm{mV}$ can be inferred from these collected data (from Jouniaux and Ishido [2012]; Allègre et al. [2010]). 


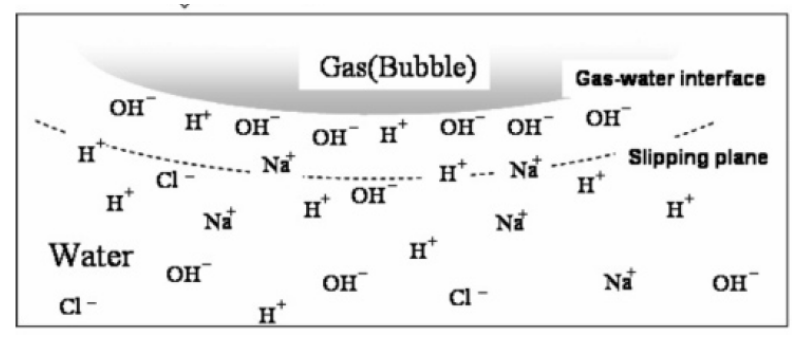

1041 Figure 15. Electric double layer at the interface gas/water (from Takahashi [2005]). The ions from water are attracted by the interface charged by $\mathrm{H}^{+}$and $\mathrm{OH}^{-}$. The zeta potential is defined as the potential on the slipping plane.

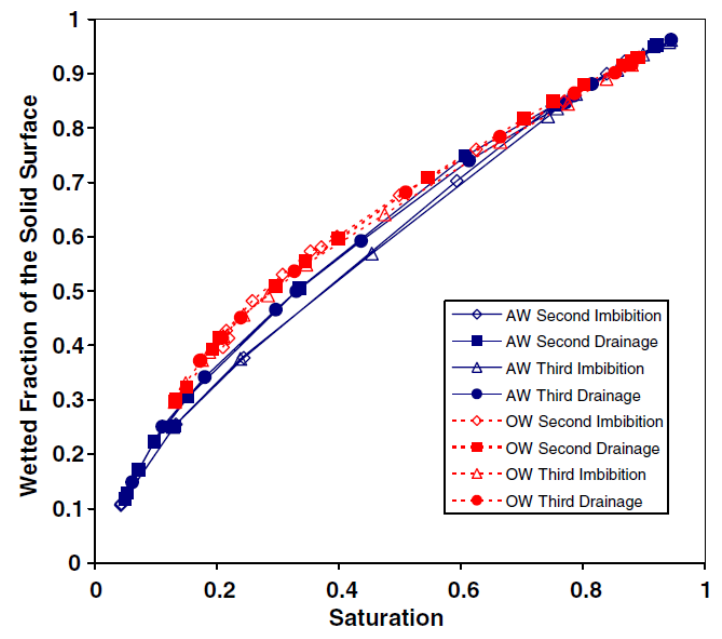

Figure 16. Interfacial areas matrix/water measured during drainage experiments by Culligan et al. [2006]. 


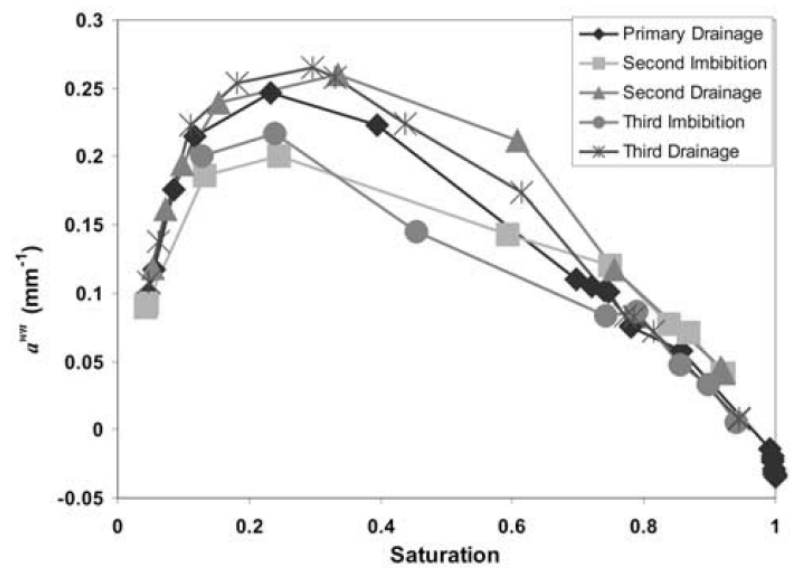

Figure 17. Interfacial area water/air measured during drainage experiments by Culligan et al. [2004]. Slightly negative values are due to numerical error in the image analysis routine or wrong identification of a small number of pixels.

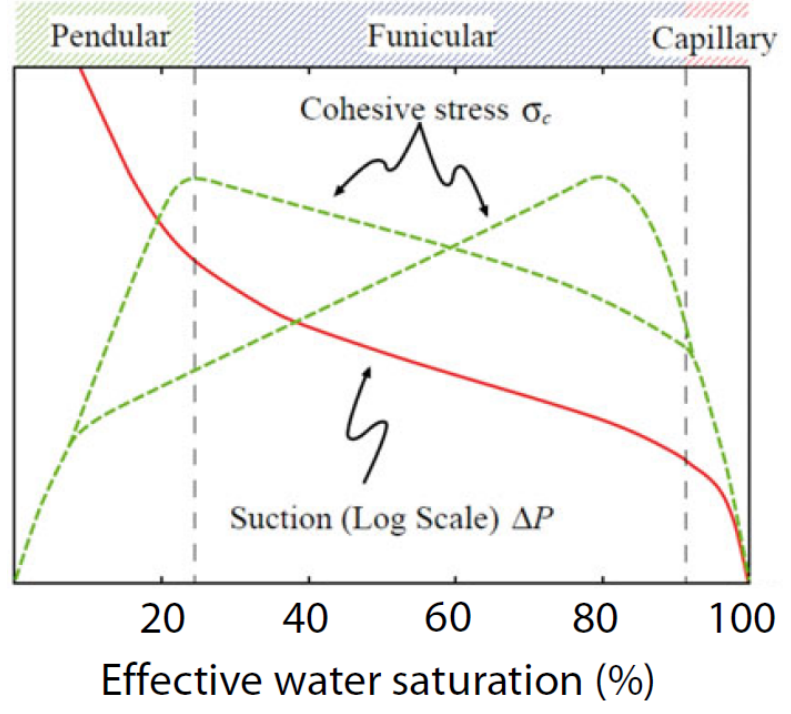

Figure 18. (Schematic variations of the cohesive stress as a function of the water saturation (modified after Mitarai and 


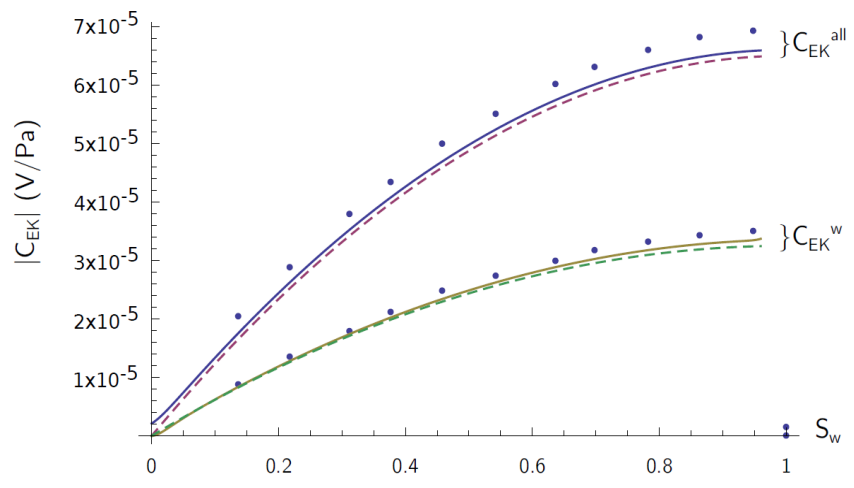

Figure 19. Streaming potential coefficient simulated in a capillary channel with an increasing air corridor in the middle of the channel when the water saturation $S_{w}$ decreases (after ?). The streaming potential coefficient is increasing with water saturation, linked to increasing fluid velocity, up to full water saturation where the SPC decreases, linked to the fact that the interface water/air does not exist anymore.

\section{$\left|C_{E K}\right|(\mathrm{V} / \mathrm{Pa})$}

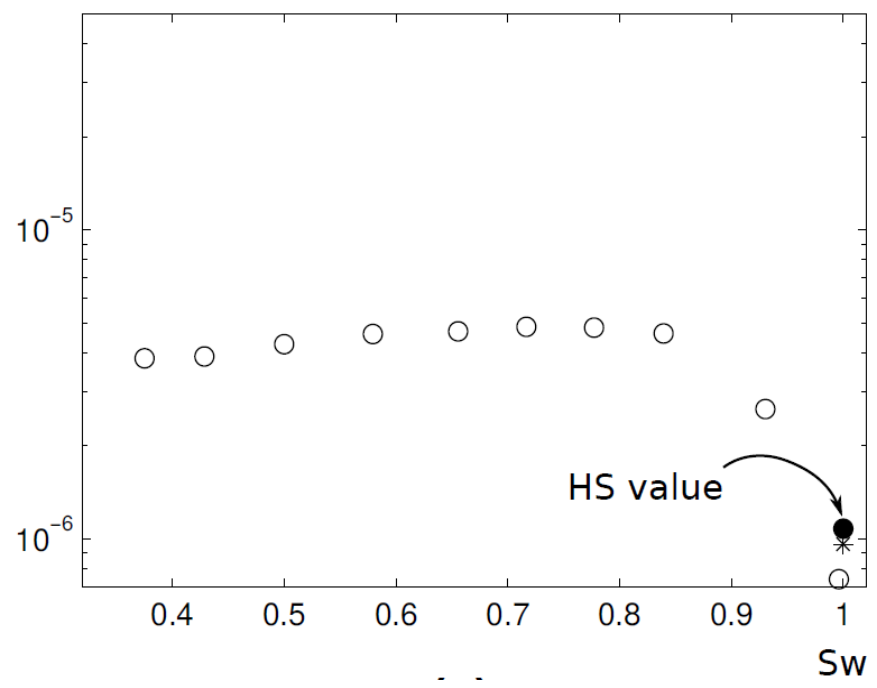

Figure 20. Streaming potential coefficient simulated in a capillary channel with an increasing entrapped bubbles on the wall of the channel for a decreasing water saturation (after Fiorentino et al. [2017]). The streaming potential coefficient shows non monotonous behaviour with water saturation, linked to a decreasing of the fluid velocity with decreasing water saturation, and to an increasing charge density and surface area of the water/air interface with decreasing water saturation. 


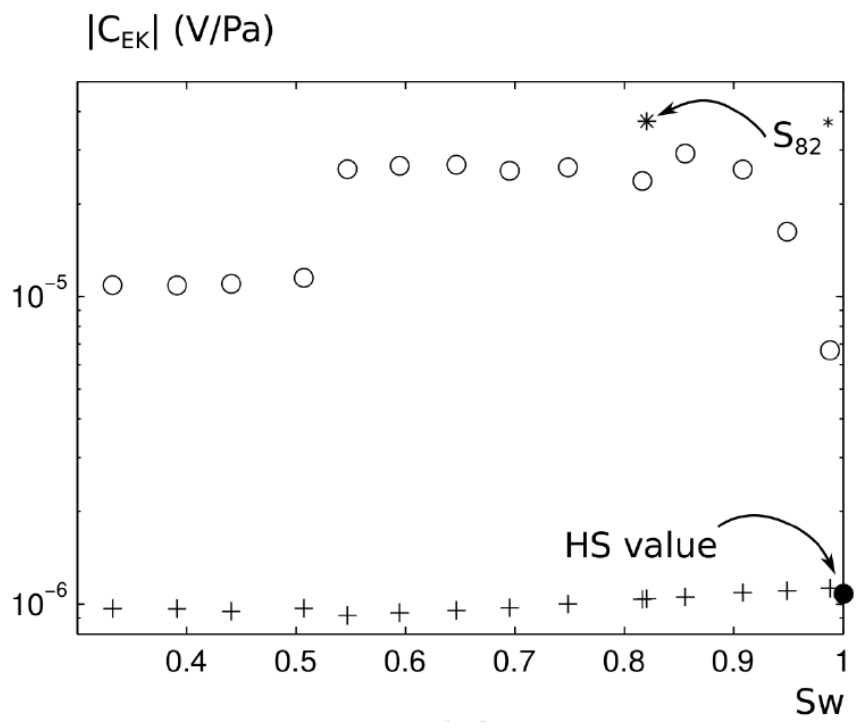

Figure 21. Streaming potential coefficient simulated in a capillary channel with increasing flowing bubbles for a decreasing water saturation (after ?). The streaming potential coefficient shows non monotonous behaviour with water saturation, linked to a decreasing of the fluid velocity with decreasing water saturation, and to an increasing charge density and surface area of the water/air interface with decreasing water saturation. The step in SPC between $55 \%$ and $50 \%$ is linked to the fact that two bubbles merge into one bubble. The dots involve a zeta potential of $-20 \mathrm{mV}$ for both matrix/water and water/air interface; the crosses are calculated with a null zeta potential at the interface water/air. 


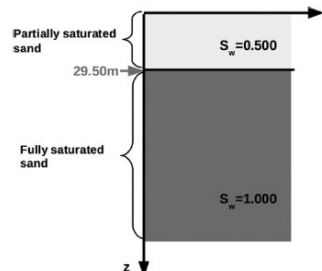

(a) PI-EM IR mean amplitude

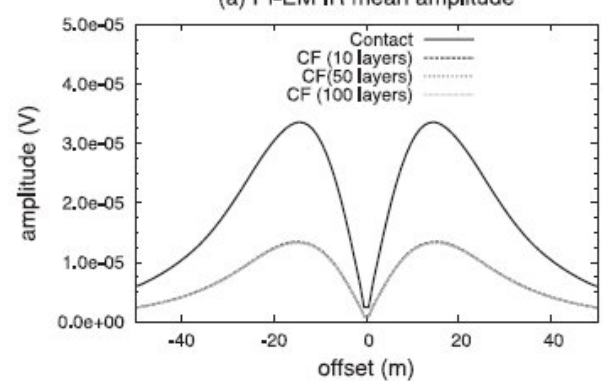

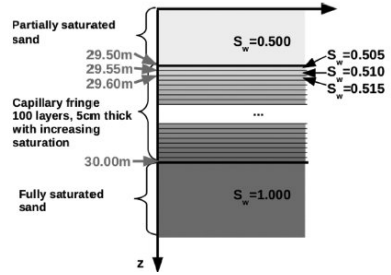

(b) S-EM IR mean amplitude

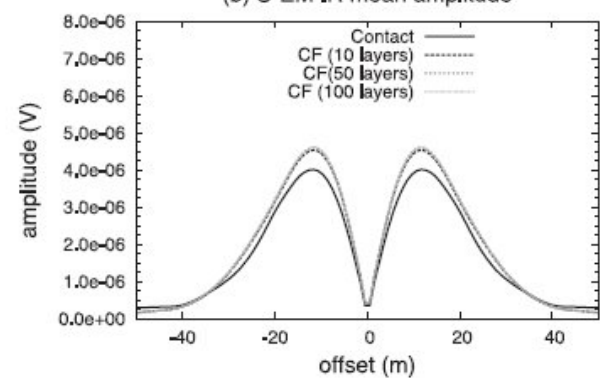

Figure 22. Comparison of the mean amplitudes of the interface response induced by compressional waves (bottom left) and shear waves (bottom right), for a sharp saturation transition (top left model) between the two considered regions and for a gradual saturation transition, as given for the capillary fringe shown in the top right model. The IR response induced by the S-wave is stronger for the capillary fringe than for the sharp saturation transition, while the IR response generated by the compressional wave is stronger for the sharp concentration transition than for the capillary fringe. 
a)

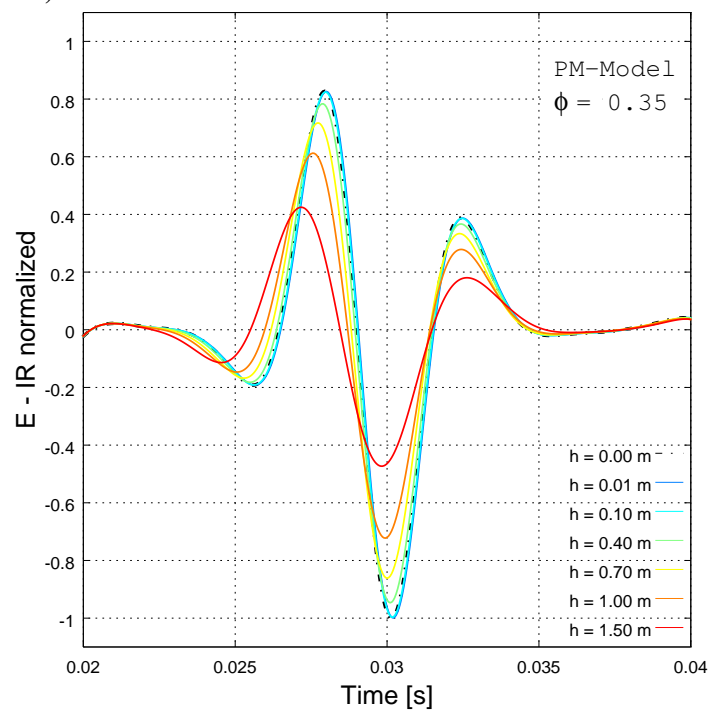

b)

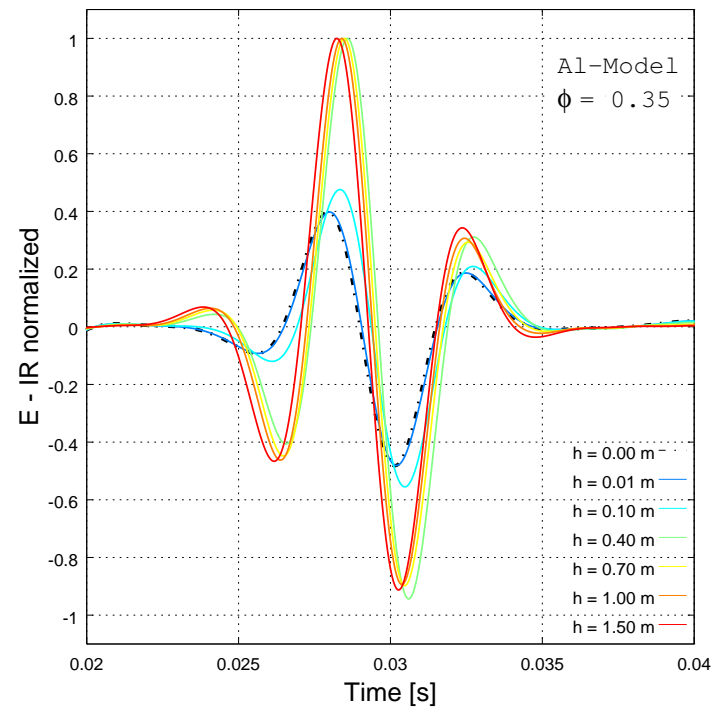

Figure 23. Electric field interface response E-IR dependence on the thickness of the contaminated layer $h$ for: a) PM-

Model and b) Al-Model (meaning Perrier-Morat model and Allègre model respectively, see Table 1). In the former, $S\left(S_{w}\right)$ is a monotonic function of water saturation, in the latter a non monotonic one. Notice that for both models the responses related to the absence of contamination $(h=0 \mathrm{~m})$ and $h=0.1 \mathrm{~m}$ are coincident, as expected. 